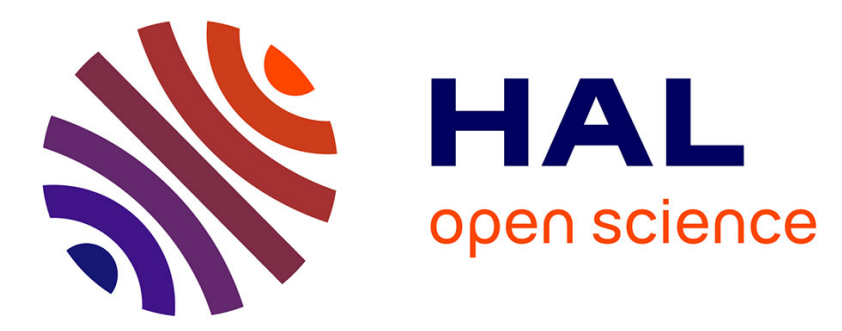

\title{
MRI-Based Electric Properties Tomography with a Quasi-Newton approach
}

\author{
Anar Rahimov, Amelie Litman, Guillaume Ferrand
}

\section{To cite this version:}

Anar Rahimov, Amelie Litman, Guillaume Ferrand. MRI-Based Electric Properties Tomography with a Quasi-Newton approach. Inverse Problems, 2017, 33 (10), pp.105004. 10.1088/1361-6420/aa7ef2 . hal-01579263

\section{HAL Id: hal-01579263 https://hal.science/hal-01579263}

Submitted on 5 Nov 2018

HAL is a multi-disciplinary open access archive for the deposit and dissemination of scientific research documents, whether they are published or not. The documents may come from teaching and research institutions in France or abroad, or from public or private research centers.
L'archive ouverte pluridisciplinaire HAL, est destinée au dépôt et à la diffusion de documents scientifiques de niveau recherche, publiés ou non, émanant des établissements d'enseignement et de recherche français ou étrangers, des laboratoires publics ou privés. 


\title{
MRI-Based Electric Properties Tomography with a Quasi-Newton approach
}

\author{
Anar Rahimov ${ }^{(1)(2)}$, Amélie Litman ${ }^{(1)}$, Guillaume Ferrand ${ }^{(3)}$ \\ (1) Aix Marseille Univ, CNRS, Centrale Marseille, Institut Fresnel, Marseille, France \\ (2) Institute of Control Systems, Azerbaijan National Academy of Sciences, \\ B. Vahabzadeh str. 9, AZ1141, Baku, Azerbaijan \\ (3) CEA-Saclay, DSM/IRFU/SACM, F-91191 Gif-sur-Yvette, France \\ E-mail: amelie.litman@fresnel.fr
}

\begin{abstract}
Magnetic resonance electric properties tomography is a non-destructive imaging modality that maps the spatial distribution of the electrical conductivity and permittivity of the human body using standard clinical magnetic resonance imaging systems. From the $B_{1}^{+}$magnetic field maps and the local form of the Maxwell equations, several schemes have been derived to provide direct approximated formulas but they suffer from instabilities. In this paper, we propose to address it as an inverse problem solved by a constrained optimization algorithm where we exploit the weak formulation of the electric Helmholtz equation and a Lagrangian approach. We derive the associated adjoint field equation and employ a Quasi-Newton minimization scheme. We also take advantage of a regularisation strategy based on geometrical a priori information for defining large zones into which the electric parameters are known to be piece-wise constant.
\end{abstract}

Keywords: inverse problem, magnetic resonance, MRI, non-linear optimization, Lagrangian approach, adjoint field, Helmholtz equation, Quasi-Newton, MR-EPT, electric properties tomography, B1 map,

\section{Introduction}

Magnetic resonance imaging (MRI) scans are nowadays routinely ordered to visualize the anatomy of the inside of the human body. In addition to these morphological maps, MRI systems can also provide quantitative mapping of the dielectric permittivity and conductivity properties inside the human body $[1,2,3]$. Such technique could help in the diagnostic of pathologies which induce an important variation of the dielectric permittivity. For example, it has been shown that cancerous tissues may have different electromagnetic values than normal tissues over a wide electromagnetic frequency spectrum [4] [5]. It could also provide a more accurate and personalized 
prediction of the Specific Absorption Rate (SAR) that is encountered in high-field and ultra high-field magnetic resonance imaging, typically above 3 Tesla (3T) [6].

The idea behind MRI-Based Electric Properties Tomography (MREPT) is to use the standard MRI system, with no extra equipment, and to employ ad-hoc postprocessing techniques to measure the distorted transmit magnetic field $B_{1}^{+}$by means of a so-called $B_{1}$ mapping technique $[7,8]$. Indeed, besides the static magnetic field $B_{0}$ from the main magnet, the radiofrequency ( $\mathrm{RF}$ ) magnetic field $B_{1}$ generated by RF coils with a sinusoidal time dependence at the Larmor frequency, reorients the magnetization of the spins so that a signal is induced and detected by the receiver coil $[9,10]$. The problem of the amplitude $\left|B_{1}^{+}\right|$mapping has been studied in a number of contributions, see for example $[11,12,13]$. Nevertheless, recovering the absolute phase of $B_{1}^{+}$seems more difficult to perform [14]. For certain coil arrangements or object geometries, it can be directly estimated [15] or the acquisition sequences must be modified specifically to that aim [14]. In this work, we will assume that we have access to the full mapping of the magnitude and the phase of the $B_{1}^{+}$field. As the distortions of the RF magnetic field are directly linked with the conductivity $\sigma$ and permittivity $\varepsilon_{r}$ of the human tissues, the measurement of such variations allows the reconstruction of the tissue's electric properties.

Several schemes have been derived to provide approximated solutions based on the local Maxwell equations. Indeed, with the $B_{1}$ mapping technique, the magnetic field $B_{1}^{+}$value is provided in a limited region of the human body. If one can derive a local inverse function linking the electric parameters to $B_{1}^{+}$, one can provide spatial distributions of these parameters inside the probed area. One possibility is to assume that the spatial variations of the complex permittivity $m=\varepsilon_{r}+j \sigma /\left(\omega \varepsilon_{0}\right)$ are small which enables to directly express them in terms of a local ratio between the Laplacian of the magnetic field and the magnetic field itself [7, 15, 16, 17, 18, 19]. Further simplifications lead to magnitude-based permittivity imaging and phase-based conductivity imaging. Unfortunately, such local inverse formulas suffer from instabilities, in particular at the boundaries from different tissues where the assumption that the spatial variations are small is no longer valid. Some weighted formulas have been introduced to compensate in parts for such effects [20]. As well, completely different schemes have been proposed, based on the introduction of a convection-reaction equation [21] linking the complex permittivity variations to the magnetic field variations. It is, however, relevant to propose new ways to recover the dielectric properties of human tissues from $B_{1}^{+}$mapping.

Following the recent works detailed in [22] and [23], we rather formulate this problem as an inverse problem where one aims at recovering the permittivity and conductivity maps from the $B_{1}^{+}$mapping. The underlying idea is that the permittivity and conductivity variations do not solely contribute to the local value of the magnetic field perturbation but instead they affect the magnetic field in a wider manner. Let us mention that similar approaches have been developed in quantitative magnetic susceptibility (QSM) where the inverse problem aims at recovering the magnetic susceptibility from magnetic fields mapping [24]. We recast the MREPT inverse 
problem into a constrained optimization algorithm, where we can incorporate the Maxwell equations in a global way as well as additional experimental knowledge, such as measurement noise and a priori information. Indeed, the data misfit at each local point can be weighted according to the signal to noise ratio which may vary from one measurement point to the other one. As well, anatomical images based on classical magnetic resonance maps combined with tissues segmentation techniques, which are now routinely used, can be incorporated into the human body description as geometrical priors.

Our work differs from [22] or [23] on several aspects. Firstly, we take advantage of the weak formulation of the electric Helmholtz equation. We thus do not need to compute several Green's tensors as done in [23] nor do we need to tackle the magnetic Helmholtz equation as done in [22]. Indeed, the magnetic Hemholtz equation involves terms in $\nabla \log m$, while the electric Helmholtz equation involves terms in $m$ only. The main drawback is that, in our case, the cost function derivation is more arduous to differentiate. It nevertheless ends up in the definition of an adjoint problem, which is the solution of the weak formulation of a similar Helmholtz equation. Secondly, contrarily to the contrast-source (CS) method employed in [23], we ensure that the Karush-KuhnTucker (KKT) conditions $[25,26]$ are always fulfilled thus computing the forward solver at each iteration. There is thus no need of the additional unknowns introduced by the CS algorithm. The computational burden associated to the forward problem is quite manageable as, at the Larmor frequency, the head size is only of few wavelengths, the wavelength being in the decimetre range. Thirdly, we take advantage of a regularisation strategy based on a priori information using image segmentation and classification in order to define the zones into which the electric parameters are known to be piece-wise constant. This enables to reduce the number of unknowns and thus render less ill-posed this inverse problem. Finally, we employ a Quasi-Newton minimization algorithm to recover the quantitative permittivity and conductivity distribution maps [27].

This paper is organized as follows. In Section 2, the forward problem is described and the finite element method which is used to compute the magnetic field distribution everywhere is briefly recalled in Section 3. The inverse problem is stated in Section 4. It is reformulated as a non-linear optimization problem with constraints whose saddlepoint conditions are formally expressed to introduce an adjoint field and the data misfit derivative. Section 5 describes how the inversion scheme is numerically implemented as it requires specific amendments. Two cases are considered: either the complex permittivity is unknown in each cell of the discretized human body or the complex permittivity is only unknown by zones, which are already predefined from anatomical maps of the human body. In Section 6, numerical results are presented to illustrate the efficiency of the proposed method. In particular, a study on the influence of the initial guess, the number of measurements, the signal to noise ratio and the zones classification is provided. Concluding remarks follow. 


\section{Definition of the configuration}

We consider a two-dimensional (2D) simplified MRI scanner configuration (Figure 1) where the relative permittivity $\varepsilon_{r}(\vec{r})$ and conductivity $\sigma(\vec{r})$ vary at the Larmor frequency within each human tissue (Table 1) [28]. A set of ideal line sources $\vec{S}\left(\vec{r} ; \vec{r}_{s}\right)=S\left(\vec{r} ; \vec{r}_{s}\right) \vec{e}_{z}$

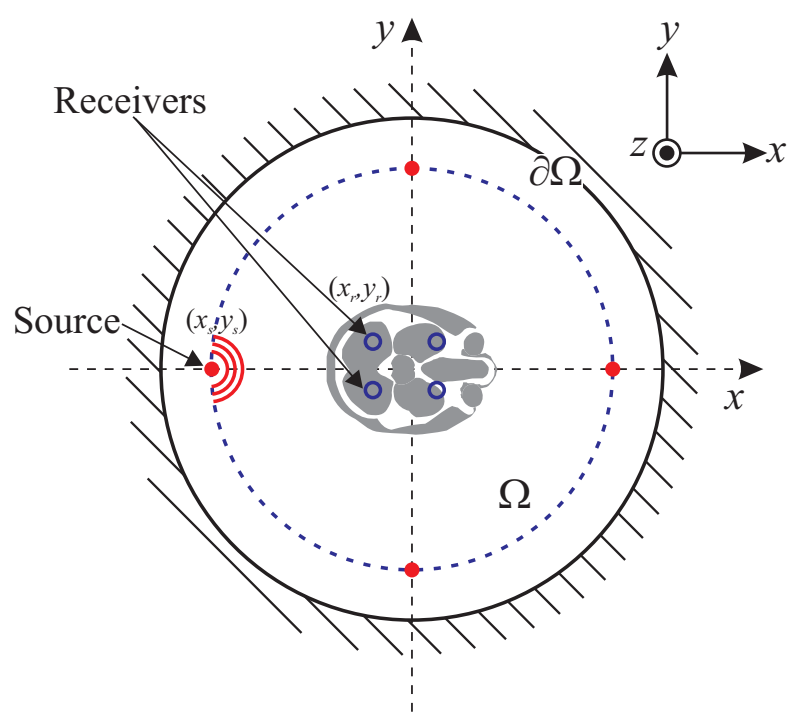

Figure 1. Geometry of the problem

positioned at $N_{s}$ different places $\vec{r}_{s}=\left(x_{s}, y_{s}\right)$ simulate the antenna elements of the RF coil [29]. The sources emit one after the other, generating an electric field $\vec{E}\left(\vec{r} ; \vec{r}_{s}\right)$ coupled to a RF magnetic field $\vec{B}\left(\vec{r} ; \vec{r}_{s}\right)$ By summing a weighted contribution of all antenna elements, one could also simulate the behaviour of more complex antenna structures, such as for example planar strip array [30]. For the sake of simplicity, we restrict ourselves to a sequential dipolar-like excitation.

Due to the invariance along the $z$ axis, the electric field is always parallel to the $z$ axis, and can be expressed as

$$
\vec{E}\left(\vec{r} ; \vec{r}_{s}\right)=E\left(x, y ; x_{s}, y_{s}\right) \vec{e}_{z}
$$

whereas

$$
\vec{B}\left(\vec{r} ; \vec{r}_{s}\right)=B_{x}\left(x, y ; x_{s}, y_{s}\right) \vec{e}_{x}+B_{y}\left(x, y ; x_{s}, y_{s}\right) \vec{e}_{y} .
$$

According to Maxwell's equation, under the $\exp (-j \omega t)$ time convention, the electromagnetic fields satisfy (see Appendix A)

$$
\nabla \times \vec{B}=-\frac{j}{\omega}\left[k^{2} m(\vec{r}) \vec{E}\left(\vec{r} ; \vec{r}_{s}\right)-\vec{S}\left(\vec{r} ; \vec{r}_{s}\right)\right] \text { and } \nabla \times \vec{E}=j \omega \vec{B}
$$

where $k$ is the wave number in air and the complex permittivity is defined by

$$
m(\vec{r})=\varepsilon_{r}(\vec{r})+j \frac{\sigma(\vec{r})}{\omega \varepsilon_{0}}
$$

where $\omega=2 \pi f, f$ being the Larmor frequency and $\varepsilon_{0}$ the vacuum permittivity. 
In the MREPT literature, several modelling approaches have been proposed. For example, in $[2,22,18]$, the Helmholtz equation for the RF magnetic field $B$ is exploited. At first glance, such a formulation could seem natural as it only involves the magnetic field. Nevertheless, it is a vectorial equation which does not fully benefit from the fact that the configuration can be considered as a two-dimensional one. Moreover, the dependency with respect to the electromagnetic properties $m(\vec{r})$ is expressed in a highly non-linear way. In [23], the electromagnetic fields are obtained by two Fredholm integral representations, involving the computation of two Green's tensors: the electric current to magnetic field tensor and the electric current to electric field tensor.

In the present work, we instead favour the Helmholtz equation for the electric field

$$
\begin{aligned}
\Delta E\left(\vec{r} ; \vec{r}_{s}\right)+k^{2} m(\vec{r}) E\left(\vec{r} ; \vec{r}_{s}\right) & =S\left(\vec{r} ; \vec{r}_{s}\right) & & \text { in } \Omega, \\
E\left(\vec{r} ; \vec{r}_{s}\right) & =0 & & \text { on } \partial \Omega .
\end{aligned}
$$

as it is a scalar equation much simpler to solve. A Dirichlet boundary condition has been expressed here but any other type of boundary condition may also apply.

Due to the relationship between the electrical field and the magnetic field, it follows that

$$
\vec{B}\left(\vec{r} ; \vec{r}_{s}\right)=\frac{1}{j \omega} \operatorname{rot} \vec{E}\left(\vec{r} ; \vec{r}_{s}\right)=\frac{1}{j \omega}\left(\frac{\partial E}{\partial y} \vec{e}_{x}-\frac{\partial E}{\partial x} \vec{e}_{y}\right) .
$$

An MRI system offers the unique situation in which the magnetic fields within the object of interest can be measured. It means that any point $\vec{r}_{r}=\left(x_{r}, y_{r}\right), r=1, \cdots, N_{r}$ within the human body can be considered as a virtual receiving antenna. Unfortunately, the MRI setup can give access to some mixed components of the RF transmit field $\vec{B}$, but not necessarily to the full components of the magnetic field itself. Let us thus focus on one of them, that is

$$
B_{1}^{+}(E)=\frac{1}{2}\left(B_{x}+j B_{y}\right)=\frac{1}{2 j \omega}\left(\frac{\partial E}{\partial y}-j \frac{\partial E}{\partial x}\right) .
$$

The previous expression is a linear expression with respect to the component $E$ of the electric field.

\section{Numerical field simulations}

For computing the electromagnetic field everywhere in space, a finite element method (FEM) is implemented [31, 32]. This method is briefly recalled here as it will be needed to correctly explain the way the inversion scheme has been implemented.

The space domain $\Omega$ is subdivided into $N_{e}$ small triangular elements $\Omega_{e}$ with a mesh generator [33] (see Figure 2). We have taken the same configuration as the one described in [15], where the human head is placed in a 7T resonant birdcage coil. The associated Larmor frequency of $298 \mathrm{MHz}$ was thus selected for performing the computations. A circular metallic shield surrounds the scene at $r=18.6 \mathrm{~cm}$ while the transmitters $\vec{r}_{s}$ are equally spaced on a circle of radius $r=15 \mathrm{~cm}$. Their number will differ depending on the considered configuration. 
Classical P1 element are used, leading to

$$
E=\sum_{e=1}^{N_{e}} \sum_{i=1}^{3} E_{e}^{i} T_{e}^{i}(\vec{r})
$$

where $\mathbb{I}_{e}(\vec{r})$ is the indicator function for $\Omega_{e}$ and the basis functions are given by

$$
T_{e}^{i}(\vec{r})=\frac{1}{2 \Delta_{e}}\left(a_{e}^{i}+b_{e}^{i} x+c_{e}^{i} y\right)
$$

where $\Delta_{e}$ is the area of $\Omega_{e}$ [31]. Once the weak form of the Helmholtz equation (5) is discretized, a linear system can be written compactly as

$$
\mathbf{K E}=\mathbf{b}
$$

where $\mathbf{K}$ is the stiffness matrix, $\mathbf{E}$ is obtained from assembling $\left\{E_{e}^{i}\right\}$ and the right-handside $\mathbf{b}$ is assembled from $\left\{b_{e}^{i}\right\}$ with

$$
b_{e}^{i}=\int_{\Omega_{e}} S\left(\vec{r} ; \vec{r}_{s}\right) T_{e}^{i}(\vec{r}) d \vec{r} \quad i=1,2,3 .
$$

The sparse linear system is solved thanks to a LU decomposition algorithm [34].

\begin{tabular}{llr}
\hline Human tissue & $\varepsilon_{r}$ & $\sigma(\mathrm{S} / \mathrm{m})$ \\
\hline Cancellous bone & 23.1 & 0.21 \\
Cortical bone & 13.4 & 0.082 \\
Cerebrum & 49.6 & 0.51 \\
Brain stem & 60.0 & 0.69 \\
Cerebellum & 59.7 & 0.97 \\
Cerebrospinal fluid & 72.7 & 2.22 \\
Eye & 69.0 & 1.51 \\
Muscle & 59.5 & 0.78 \\
Spinal & 36.9 & 0.42 \\
\hline
\end{tabular}

Table 1. Description of the various brain constituents and their dielectric properties at $298 \mathrm{MHz}$, the Larmor frequency at $7 \mathrm{~T}$.

An example of a permittivity map distribution within a brain slice can be seen in Figure 3 while the associated RF magnetic $B_{1}^{+}$field is visible in Figure 4.

\section{Inverse problem formulation}

The inverse problem is stated as finding the permittivity distribution corresponding to a measured $B_{1}^{+, \text {meas }}$ field distribution. The difficulty here is twofold: (i) the value is obtained everywhere within each voxel of the brain leading to a large number of virtual receivers and (ii) this quantity is a by-product of the electrical field which requires an extra step in our simulation procedure which relies on the electrical field propagation equation. 


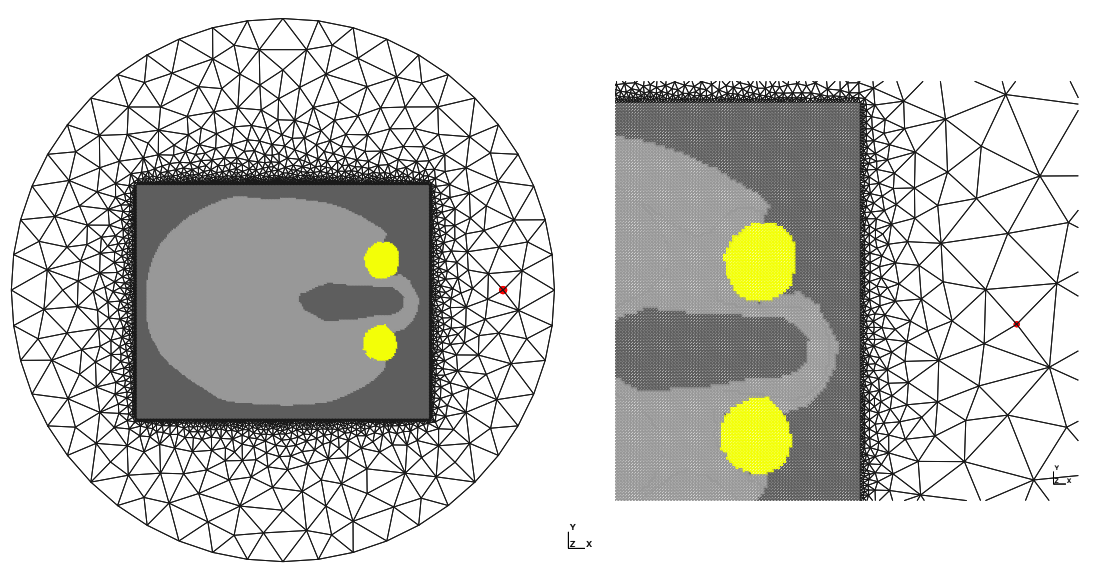

Figure 2. Mesh distribution in the cavity and the brain, with approximatively 34000 node points and 68000 triangles. In the brain area, the mesh length is of $0.1 \mathrm{~cm}$ as shown on the right in the excerpt. The mesh is conformal to the external geometry of the head, as well as the eyes and the nasal cavity. The red dot indicates one position for the transmitter.

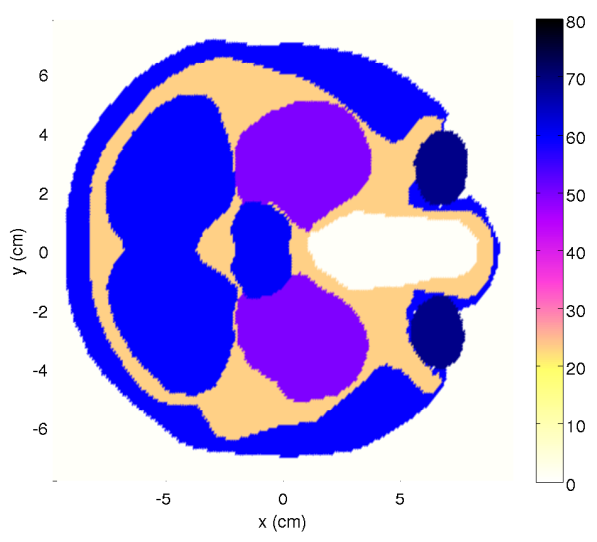

(a) Relative permittivity $\varepsilon_{r}=\operatorname{Re}(m)$

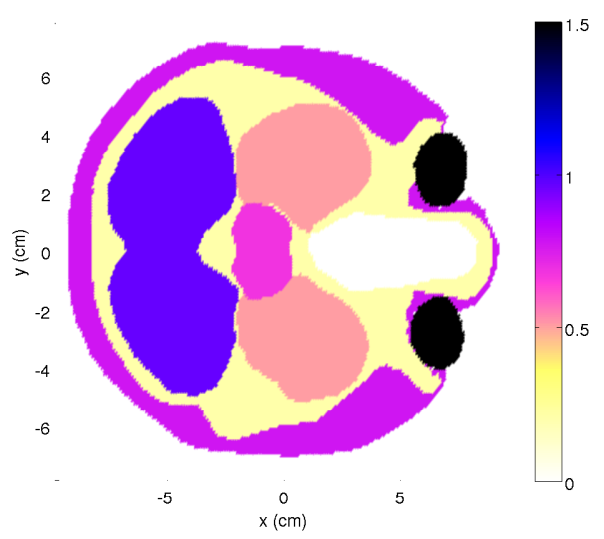

(b) Conductivity $\sigma=\omega \varepsilon_{0} \operatorname{Im}(m)$

Figure 3. Electromagnetic parameters distribution within a slice of the brain at $298 \mathrm{MHz}$.

\subsection{Constrained optimization formulation}

This problem can be recasted as a non-linear optimization problem with constraints. Indeed, from these measurements $B_{1}^{+, \text {meas }}$, we define a cost function $\mathcal{F}$ which corresponds to the discrepancy between the measured quantities and the simulated ones at any point in space,

$$
\begin{aligned}
\mathcal{F} & =\sum_{s=1}^{N_{s}} \mathcal{F}_{s}=\frac{\omega^{2}}{2 N_{s}} \sum_{s=1}^{N_{s}} \sum_{r=1}^{N_{r}}\left|B_{1}^{+}(E)\left(\vec{r}_{r} ; \vec{r}_{s}\right)-B_{1}^{+, \text {meas }}\left(\vec{r}_{r} ; \vec{r}_{s}\right)\right|^{2} \\
& =\frac{\omega^{2}}{2 N_{s}} \sum_{s=1}^{N_{s}} \sum_{r=1}^{N_{r}} \int_{\Omega}\left|B_{1}^{+}(E)\left(\vec{r} ; \vec{r}_{s}\right)-B_{1}^{+, \text {meas }}\left(\vec{r} ; \vec{r}_{s}\right)\right|^{2} \delta\left(\vec{r}-\vec{r}_{r}\right) d \vec{r} .
\end{aligned}
$$



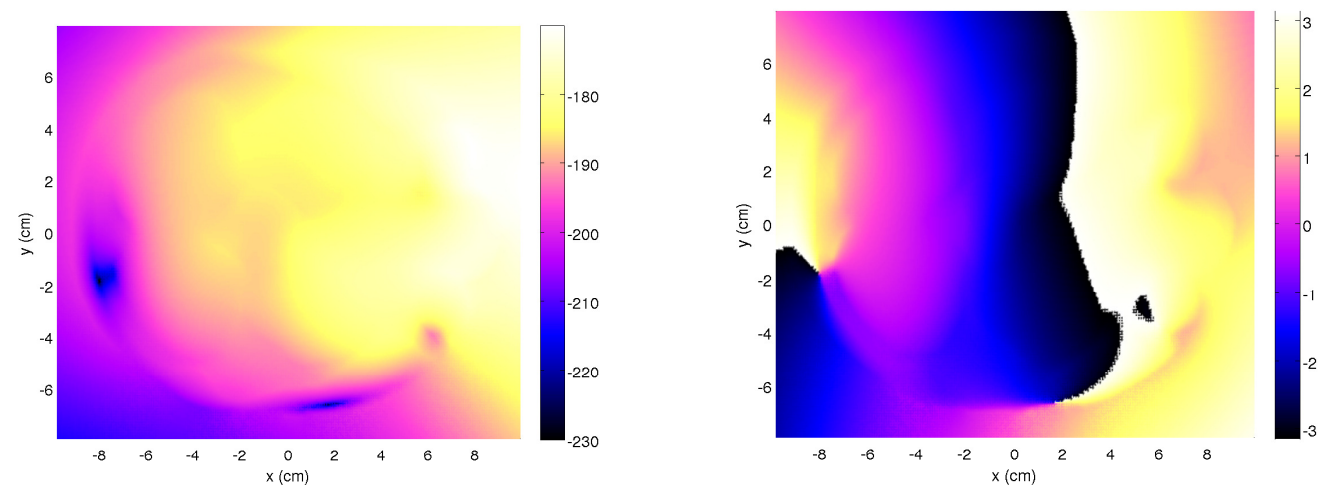

Figure 4. Amplitude in $\mathrm{dB}$ (left) and phase in radian (right) of the $B_{1}^{+}$magnetic field distribution within the brain, at $298 \mathrm{MHz}$.

The sought-after permittivity distribution must minimize this cost function while the associated electromagnetic field must satisfy the Helmholtz equation. A Lagrangian formalism is thus introduced to incorporate these constraints

$$
\mathcal{L}(E, U, m)=\sum_{s=1}^{N_{s}} \mathcal{L}_{s}\left(E\left(\cdot ; \vec{r}_{s}\right), U\left(\cdot ; \vec{r}_{s}\right), m\right)
$$

with

$$
\mathcal{L}_{s}\left(E\left(\cdot ; \vec{r}_{s}\right), U\left(\cdot ; \vec{r}_{s}\right), m\right)=\mathcal{F}_{s}\left(E\left(\cdot ; \vec{r}_{s}\right)\right)+\operatorname{Re} \mathcal{H}_{s}\left(E\left(\cdot ; \vec{r}_{s}\right), U\left(\cdot ; \vec{r}_{s}\right), m\right)
$$

where the Helmholtz constraints is expressed with

$$
\mathcal{H}_{s}(E, U, m)=\left\langle\Delta E\left(\cdot ; \vec{r}_{s}\right)+k^{2} m(\cdot) E\left(\cdot ; \vec{r}_{s}\right)-S\left(\cdot ; \vec{r}_{s}\right) \mid U\left(\cdot ; \vec{r}_{s}\right)\right\rangle_{\Omega} .
$$

Here, $U\left(\cdot ; \vec{r}_{s}\right)$ are the Lagrange multipliers and $\langle u \mid v\rangle_{\Omega}=\int_{\Omega} u \bar{v}$ is the classical scalar product on $\Omega$.

In order to be able to extract some information related to the dielectric permittivity maps from the measured quantities, it is of interest to establish the derivative of the previous cost function with respect to $m$. For this, we construct the Karush-KuhnTucker (KKT) conditions [25, 26] to find the saddle-point, which requires that

$$
\frac{\partial \mathcal{L}}{\partial E\left(\cdot ; \vec{r}_{s}\right)}=\frac{\partial \mathcal{L}}{\partial U\left(\cdot ; \vec{r}_{s}\right)}=0 . \quad \forall E\left(\cdot ; \vec{r}_{s}\right), U\left(\cdot ; \vec{r}_{s}\right)
$$

At that saddle-point, it is well-known that

$$
\frac{\partial \mathcal{L}}{\partial m}=\frac{\partial \mathcal{F}}{\partial m} \text {. }
$$

\subsection{Derivative expressions with respect to the fields}

It can be shown (see Appendix B.1) that the derivative of the Lagrangian functional with respect to $U\left(\cdot ; \vec{r}_{s}\right)$ is given by

$$
\lim _{t \rightarrow 0} \frac{\mathcal{L}(E, U+t v, m)-\mathcal{L}(E, U, m)}{t}=\operatorname{Re}\left\langle\Delta E+k^{2} m E-S\left(\cdot ; \vec{r}_{s}\right) \mid v\right\rangle_{\Omega} .
$$


Therefore, at the saddle-point, we need to satisfy the classical Helmholtz equation for the electrical field.

The derivative with respect to $E\left(\cdot, \vec{r}_{s}\right)$ is less straight-forward (see Appendix B.2). Nevertheless, as the function $B_{1}^{+}$is linear, we have

$$
\begin{aligned}
\lim _{t \rightarrow 0} \frac{\mathcal{L}(E+t v, U, m)-\mathcal{L}(E, U, m)}{t}=\operatorname{Re}\left\langle\Delta v+k^{2} m v \mid U\left(\cdot ; \vec{r}_{s}\right)\right\rangle_{\Omega} \\
+\operatorname{Re} \frac{\omega^{2}}{N_{s}} \sum_{r=1}^{N_{r}} \int_{\Omega} \overline{\left[B_{1}^{+}(E)\left(\vec{r} ; \vec{r}_{s}\right)-B_{1}^{+, \text {meas }}\left(\vec{r} ; \vec{r}_{s}\right)\right]} B_{1}^{+}(v) \delta\left(\vec{r}-\vec{r}_{r}\right) d \vec{r} .
\end{aligned}
$$

At the saddle-point, based from (20), the KKT conditions (17) and the first Green formula, we obtain while noting $P=\bar{U}$,

$$
\begin{aligned}
\int_{\Omega}[-\nabla v(\vec{r}) \nabla & \left.P\left(\vec{r} ; \vec{r}_{s}\right)+k^{2} m(\vec{r}) P\left(\vec{r} ; \vec{r}_{s}\right) v(\vec{r})\right] d \vec{r} \\
= & -\frac{\omega^{2}}{N_{s}} \sum_{r=1}^{N_{r}} \int_{\Omega} \overline{\left[B_{1}^{+}(E)-B_{1}^{+, \text {meas }}\right]}\left(\vec{r} ; \vec{r}_{s}\right) B_{1}^{+}(v) \delta\left(\vec{r}-\vec{r}_{r}\right) d \vec{r} .
\end{aligned}
$$

The left-hand side of this equation is nothing but the left-hand side of the weak form of the Helmholtz equation (5). This is a classical result as the Helmholtz equation is autoadjoint in the present configuration. The right-hand side differs from the one dictating the electrical field behaviour. In (21), the source distribution is weighted according to the local discrepancy at each virtual receiver point between the measured magnetic field $B_{1}^{+, \text {meas }}$ and the simulated one $B_{1}^{+}$. Unfortunately, due to the $B_{1}^{+}(v)$ term, which is defined in (8), it seems not possible to derive a strong formulation similar to (5) for (21). This is not an issue here as we are employing the finite element method which definitely requires the weak formulation. Thus (21) is perfectly adapted for our simulation tool in order to compute $P\left(\cdot ; \vec{r}_{s}\right)$. The specific implementation for computing $B_{1}^{+}(v)$ will be detailed in Section 5.1.

\subsection{Derivative expressions with respect to the permittivity}

It can be shown (see Appendix C) that the derivative of the Lagrangian functional with respect to $m$ is given by

$\lim _{t \rightarrow 0} \frac{\mathcal{L}(E, U, m+t v)-\mathcal{L}(E, U, m)}{t}=\operatorname{Re}\left\langle k^{2} \sum_{s=1}^{N_{s}} \bar{E}\left(\cdot ; \vec{r}_{s}\right) \bar{P}\left(\cdot ; \vec{r}_{s}\right) \mid v\right\rangle_{\Omega}$.

Using the definition of complex derivatives of real-valued functions [35, 36], it follows from (22) that the gradient of the cost functional is

$$
\nabla_{m} \mathcal{F}=k^{2} \sum_{s=1}^{N_{s}} \bar{E}\left(\cdot ; \vec{r}_{s}\right) \bar{P}\left(\cdot ; \vec{r}_{s}\right)
$$

where the electrical field $E$ satisfies the Helmholtz equation (5) with some source terms distributed at the transmitters locations and the adjoint field $P$ satisfies a similar equation (21) with some source terms which are distributed at each virtual receiver point. 


\subsection{Regularization strategy}

The first output of an MRI procedure is a qualitative mapping of the human body. It thus automatically provides some a priori knowledge on the geometrical features of the head worth exploiting in the inverse problem in order to reduce its ill-posedness. Therefore, advanced image processing tools can be applied to the MRI image for segmenting and classifying the various components of the brain. The resulting zones $\Omega_{g}$ will be considered as spatial priors. It is not required for a zone to be simply connected, i.e., made of adjacent elements.

Depending on the regularization choice, the unknown permittivity distribution is interpolated and assumed to be piece-wise constant either on each triangle $\Omega_{e}$ (for an element-based inversion without a priori knowledge) or on each zone $\Omega_{g}$ (for a zonebased inversion with a priori geometrical knowledge),

$$
m(\vec{r})=\sum_{e=1}^{N_{e}} m_{e} \mathbb{I}_{e}(\vec{r}) \quad \text { or } \quad m(\vec{r})=\sum_{g=1}^{N_{g}} m_{g} \mathbb{I}_{g}(\vec{r}) .
$$

We can express the derivatives with respect to each set of unknowns, using the chain rule derivation,

$$
\nabla_{m_{\bullet}} \mathcal{F}=k^{2} \sum_{s=1}^{N_{s}} \int_{\Omega \bullet} \bar{E}\left(\vec{r} ; \vec{r}_{s}\right) \bar{P}\left(\vec{r} ; \vec{r}_{s}\right) d \vec{r},
$$

with $\bullet=e$ or $g$ depending on the regularization strategy.

\section{Numerical implementation of the inversion scheme}

In the following, we will describe how the adjoint field has been computed as well as the minimization scheme that has been implemented to obtain the numerical results shown in Section 6.

\subsection{Adjoint field computation}

Due to the specific form of the adjoint equation, it is worth detailing the way the righthand-side term of (21) is computed. As in (9), let us assume that

$$
P=\sum_{e=1}^{N_{e}} \sum_{i=1}^{3} P_{e}^{i} T_{e}^{i}(\vec{r})
$$

Since $T_{e}^{i}(\vec{r})$ vanishes outside of $\Omega_{e}$, the right-hand-side of (21) is reduced to, when $v=P$,

$$
\begin{array}{r}
-\frac{\omega^{2}}{N_{s}} \sum_{r=1}^{N_{r}} \int_{\Omega} \overline{\left[B_{1}^{+}(E)-B_{1}^{+, \text {meas }}\right]}\left(\vec{r} ; \vec{r}_{s}\right) B_{1}^{+}\left(\sum_{e=1}^{N_{e}} \sum_{i=1}^{3} P_{e}^{i} T_{e}^{i}(\vec{r})\right) \delta\left(\vec{r}-\vec{r}_{r}\right) d \vec{r} \\
=-\frac{\omega^{2}}{N_{s}} \sum_{r=1}^{N_{r}} \sum_{i=1}^{3} P_{e}^{i} \int_{\Omega_{e}} \overline{\left[B_{1}^{+}(E)-B_{1}^{+, \text {meas }}\right]}\left(\vec{r} ; \vec{r}_{s}\right) B_{1}^{+}\left(T_{e}^{i}(\vec{r})\right) \delta\left(\vec{r}-\vec{r}_{r}\right) d \vec{r} .
\end{array}
$$


taking into account that $B_{1}^{+}$is a linear operator. Following the Ritz method [31], we derivate with respect to $P_{e}^{i}$ to obtain the local right-hand-side term which will appear in the linear system,

$$
\begin{aligned}
\operatorname{RHS}_{e}^{i} & =-\frac{\omega^{2}}{N_{s}} \sum_{r=1}^{N_{r}} \int_{\Omega_{e}} \overline{\left[B_{1}^{+}(E)-B_{1}^{+, \text {meas }}\right]}\left(\vec{r} ; \vec{r}_{s}\right) B_{1}^{+}\left(T_{e}^{i}(\vec{r})\right) \delta\left(\vec{r}-\vec{r}_{r}\right) d \vec{r} \\
& =-\frac{\omega^{2}}{N_{s}} \sum_{r=1}^{N_{r}} \int_{\Omega_{e}} \overline{\left[B_{1}^{+}(E)-B_{1}^{+, \text {meas }}\right]}\left(\vec{r} ; \vec{r}_{s}\right) \frac{1}{2 j \omega}\left[\frac{\partial T_{e}^{i}}{\partial y}-j \frac{\partial T_{e}^{i}}{\partial x}\right](\vec{r}) \delta\left(\vec{r}-\vec{r}_{r}\right) d \vec{r} .
\end{aligned}
$$

Due to the choice of the basis functions (see (10)), we have

$$
\frac{\partial T_{e}^{i}}{\partial x}=\frac{b_{e}^{i}}{2 \Delta_{e}} \quad \text { and } \quad \frac{\partial T_{e}^{i}}{\partial y}=\frac{c_{e}^{i}}{2 \Delta_{e}} .
$$

When we replace into the right-hand-side, we obtain

$$
\mathrm{RHS}_{e}^{i}=-\frac{\omega}{4 j N_{s}} \frac{c_{e}^{i}-j b_{e}^{i}}{\Delta_{e}} \sum_{r=1}^{N_{r}} \int_{\Omega_{e}} \overline{\left[B_{1}^{+}(E)-B_{1}^{+, \text {meas }}\right]}\left(\vec{r} ; \vec{r}_{s}\right) \delta\left(\vec{r}-\vec{r}_{r}\right) d \vec{r} .
$$

The $B_{1}^{+}(E)$ magnetic field value is simulated at the virtual receiver points $\vec{r}_{r}$ via an interpolation based on the values at the adjacent nodes points. To compute the adjoint field, we must solve the following sparse linear system

$$
\text { K P }=\text { RHS }
$$

where $\mathbf{K}$ is the same stiffness matrix as in (11) and $\mathbf{R H S}=\sum_{e}\left\{\mathrm{RHS}_{e}\right\}$ is assembled from the local right-hand-side terms.

Contrarily to (5), in (21), there are a lot of points in space where the right-handside term is taking non-null values. In the forward problem computations, the number of transmitters $N_{s}$ is limited and thus we solve sequentially the linear system (11) for each transmitter location $\vec{r}_{s}$. In the adjoint problem, there are a large number of virtual receivers $N_{r}$, maybe as many as the number of elements in the MRI image. Computationally speaking, it is worthless to compute (27) for each virtual receiver $\vec{r}_{r}$ separately and sum them up afterwards. Instead, we gather all the contributions as expressed in (26) to obtain the right-hand-side for a given transmitter $\vec{r}_{s}$. We thus have for the adjoint problem as many right-hand-sides as there are transmitters.

\subsection{Minimization algorithm}

Once the adjoint and total fields have been computed, the gradient is easily derived thanks to (25), where the integral has been approximated with a mid-point rule. The unknown parameters $\mathbf{m}=\left\{m_{\bullet}\right\}$ are weighted according to the area of $\Omega_{\bullet}$, with $\bullet=e$ or $g$ depending on the regularization strategy.

An iterative scheme based on a Quasi-Newton algorithm is implemented in order to estimate the minimum of (13)

$$
\mathbf{m}^{(n+1)}=\mathbf{m}^{(n)}+\alpha^{(n)} \mathbf{d}^{(n)} .
$$


The descent direction $\mathbf{d}^{(n)}$ is obtained via a limited-memory Broyden-Fletcher-GoldfarbShanno (LM-BFGS) algorithm [25, 37, 38]

$$
\mathbf{d}^{(n)}=-\mathbf{H}^{(n)}\left\{\nabla_{m} \mathcal{F}\left(\mathbf{m}^{(n)}\right)\right\}
$$

where $\mathbf{H}^{(n)}$ is an approximation of the inverse of the Hessian. The limited-version is of interest here in particular when we try to estimate the values of the permittivity in each triangular cell as in that case, the number of unknowns $N_{e}$ is large and the approximated Hessian matrices can be dense. An additional constraint is introduced when selecting the descent direction. Indeed, such a descent direction is projected in order to ensure that $\operatorname{Re}(\mathbf{m}) \geq 1$ and $\operatorname{Im}(\mathbf{m}) \geq 0$. The bound constraints are then ensured with an active set algorithm fully detailed in [37]. The algorithm stops if: (i) the maximum number of iterations $N$ is reached, (ii) the relative variation of the cost function between two subsequent iterations is lower than a given threshold $\eta$, or (iii) the norm of the gradient is smaller than a given threshold $\xi$.

\section{Numerical results}

In this section, we present numerical results to illustrate the efficiency of the inversion algorithm proposed in the previous sections. To that end, we have played with three parameters: (i) the initial guess, (ii) the noise which is added to the simulated fields and (iii) the number of transmitters.

\subsection{Numerical configuration}

The configuration was partially described in Section 3. The $B_{1}^{+}$mapping is performed by virtual receivers which are equally spaced with a spacing of $\Delta x=0.66 \mathrm{~cm}$ and $\Delta y=0.52 \mathrm{~cm}$, slightly larger than the mesh length inside the brain. They are only positioned within the head, leading to $N_{r}=597$. There is thus no correspondence between the finite element mesh and the virtual receiving points locations. The number $N_{s}$ of transmitters varies between 1 and 32 .

In the element-based inversion, the inverse problem is under-determined as the number of acquired data is very low with respect to the number of unknowns (here $\left.N_{e} \approx 62000\right)$. In the zone-based inversion, the inverse problem is over-determined as $N_{g}=5$. Indeed, in the considered brain slice, only the following human tissues are searched for: Cancellous bone, Cerebrum, Brain stem, Cerebellum and Muscle. We have arbitrarily decided that the nasal cavity, the eyes as well as the surrounding medium are already known and their permittivities are thus kept constant during the inversion procedure.

Numerical experiments were carried out to minimize the functional defined in (13) under the constraints (5)-(6) using the LM-BFGS algorithm. In all the following examples, the initial guess $\mathbf{m}^{(0)}$ is chosen as described in Section 6.2. For the stopping criteria, we have selected $N=100, \eta \approx 0.210^{-8}$ (corresponding to an average tolerance 
value) and $\xi=10^{-8}\left|\nabla \mathcal{F}\left(\mathbf{m}^{(0)}\right)\right|$. The true distribution map $\mathbf{m}^{\text {true }}$ is the one described in Figure 3.

\subsection{Input parameters}

The initial guess is randomly chosen, with a Gaussian distribution centred on a mean value and a given variance $v_{m}$ for each elementary unknown,

$$
\mathbf{m}^{(0)}=\mathbf{m}^{\text {true }}+\left\{\mathcal{N}\left(0, v_{m}\right)\right\}+j\left\{\mathcal{N}\left(0, v_{m}\right)\right\}
$$

The value of the variance $v_{m}$ is not directly given but provided in $\mathrm{dB}$ via the input parameter Signal-to-Noise Ratio $\mathrm{SNR}_{m}$, with the following correspondence

$$
v_{m}=\frac{1}{2} 10^{-\mathrm{SNR}_{m} / 10} P_{m}
$$

where $P_{m}=<\left|\mathbf{m}^{\text {true }}\right|^{2}>$ is the average power value.

The simulated fields are as well corrupted by additive Gaussian white noise. The measurement fields which are thus considered as input data to the inversion algorithm are described by

$$
\mathbf{B}_{1}^{+, \text {meas }}=\mathbf{B}_{1}^{+}\left(\mathbf{m}^{\text {true }}\right)+\left\{\mathcal{N}\left(0, v_{B}\right)\right\}+j\left\{\mathcal{N}\left(0, v_{B}\right)\right\}
$$

The value of the variance $v_{B}$ is provided in $\mathrm{dB}$ via the input parameter Signal-to-Noise Ratio $\mathrm{SNR}_{B}$, with the following correspondence

$$
v_{B}\left(\vec{r}_{s}\right)=\frac{1}{2} 10^{-\mathrm{SNR}_{B} / 10} P_{B}\left(\vec{r}_{s}\right)
$$

with $P_{B}\left(\vec{r}_{s}\right)=<\left|B_{1}^{+}\left(\cdot ; \vec{r}_{s}\right)\right|^{2}>$ the average power value of the $B_{1}^{+}$magnetic field. It is worth mentioning that $\mathrm{SNR}_{B}=20 \log _{10} \mathrm{SNR}_{M R I}$, where $\mathrm{SNR}_{M R I}$ is the signal-to-noise ratio traditionally provided in MRI systems [39].

Several numerical tests have been performed with $\mathrm{SNR}_{B}$ ranging from $20 \mathrm{~dB}$ to $100 \mathrm{~dB}$, the latter one corresponding to a case with no noise. In practice, we do not encounter $\mathrm{SNR}_{B}$ values which cover such a large range (see for example the $\mathrm{SNR}_{B}$ values estimated with actual 7T setups [40] which are of the order of 40 to $70 \mathrm{~dB}$ ). Nevertheless, we have explored it in order to understand the limitations and robustness aspects of the proposed inversion scheme.

\subsection{Inversion outputs}

As usual, the evolution of the cost function (Figure 5) is the key parameter in order to control the behaviour of the inversion algorithm. Each time the algorithm stops, we also plot the final reconstructed complex permittivity map $\mathbf{m}^{(*)}$, as shown in Figure 6 .

Unfortunately, while looking at Figure 6, it is rather difficult to distinguish the differences between the final reconstructed maps, even if they were generated from two different initial guesses. Therefore, we also compute the discrepancy between the final reconstructed complex permittivity maps $\mathbf{m}^{(*)}$ and the true one $\mathbf{m}^{\text {true }}$. In order to have 

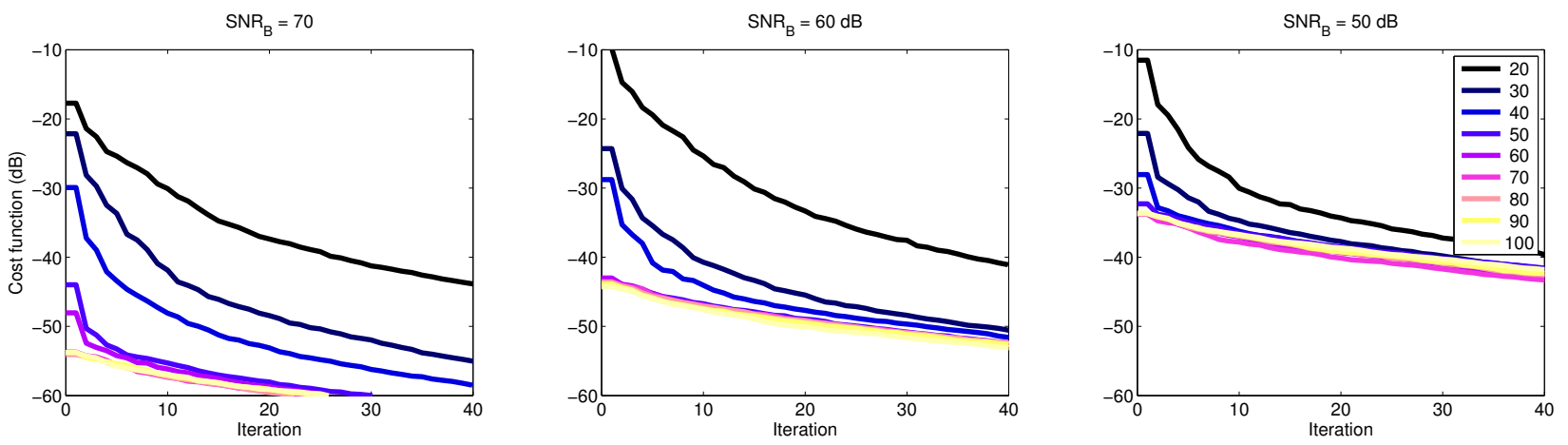

Figure 5. Cost function $\mathcal{F}$ evolution along the iterations for various $\mathrm{SNR}_{m}$ and $\mathrm{SNR}_{B}$ $\left(N_{s}=1\right.$, element-based inversion).

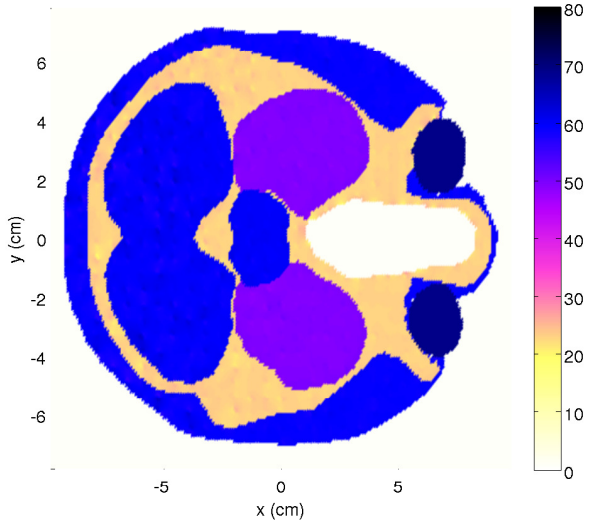

(a) $\mathrm{SNR}_{B}=60 \mathrm{~dB}, \mathrm{SNR}_{m}=40 \mathrm{~dB}$

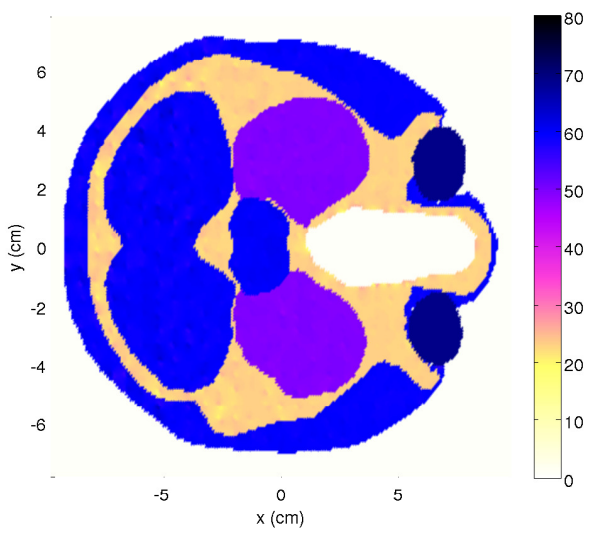

(c) $\mathrm{SNR}_{B}=60 \mathrm{~dB}, \mathrm{SNR}_{m}=60 \mathrm{~dB}$

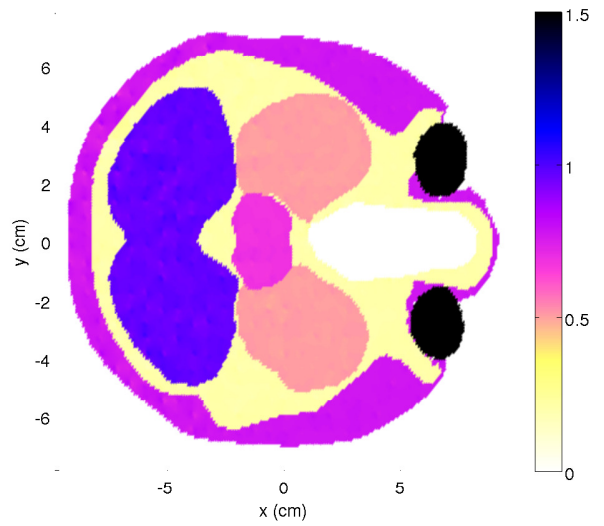

(b) $\mathrm{SNR}_{B}=60 \mathrm{~dB}, \mathrm{SNR}_{m}=40 \mathrm{~dB}$

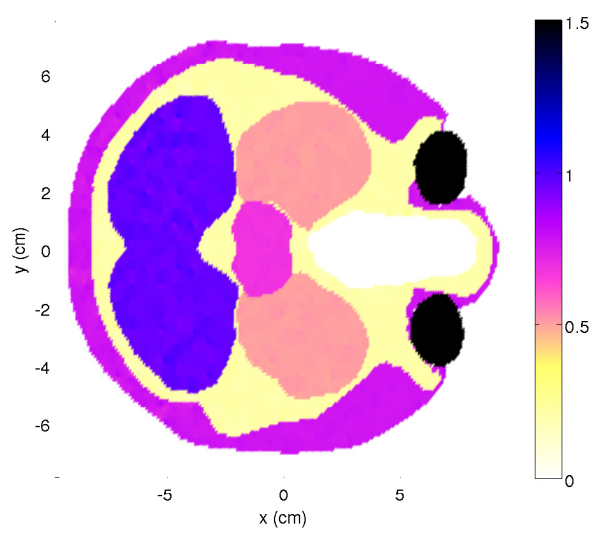

(d) $\mathrm{SNR}_{B}=60 \mathrm{~dB}, \mathrm{SNR}_{m}=60 \mathrm{~dB}$

Figure 6. Final reconstructions for two different initial guesses: (a)(c) $\varepsilon_{r}=\operatorname{Re}\left(\mathbf{m}^{(*)}\right)$ and (b)(d) $\boldsymbol{\sigma}=\omega \varepsilon_{0} \operatorname{Im}\left(\mathbf{m}^{(*)}\right)$. ( $N_{s}=1$, element-based inversion).

a more comprehensible comparison of the influence of the various input parameters, we 
plot the average values $\langle\mathbf{m}\rangle=\left\{\langle m\rangle_{g}\right\}$ per zone, which are defined by

$$
\langle m\rangle_{g}=\frac{1}{N_{e}^{g}} \sum_{e=1}^{N_{e}} m_{e} \mathbb{I}_{g}\left(\vec{r}_{e}\right) \quad \text { or } \quad\langle m\rangle_{g}=m_{g}
$$

depending if one is considering the element-based or the zone-based inversion version. $N_{e}^{g}$ corresponds to the number of elements which are indeed in the $g$-th zone,

$$
N_{e}^{g}=\sum_{e=1}^{N_{e}} \mathbb{I}_{g}\left(\vec{r}_{e}\right)
$$

The relative quantities

$$
\begin{aligned}
& \mathbf{b}^{\varepsilon_{r}}=\left\langle\frac{\boldsymbol{\varepsilon}_{r}^{(*)}-\boldsymbol{\varepsilon}_{r}^{\text {true }}}{\boldsymbol{\varepsilon}_{r}^{\text {true }}}\right\rangle=\left\langle\frac{\operatorname{Re}\left(\mathbf{m}^{(*)}-\mathbf{m}^{\text {true }}\right)}{\operatorname{Re}\left(\mathbf{m}^{\text {true }}\right)}\right\rangle \\
& \mathbf{b}^{\sigma}=\left\langle\frac{\boldsymbol{\sigma}^{(*)}-\boldsymbol{\sigma}^{\text {true }}}{\boldsymbol{\sigma}^{\text {true }}}\right\rangle=\left\langle\frac{\operatorname{Im}\left(\mathbf{m}^{(*)}-\mathbf{m}^{\text {true }}\right)}{\operatorname{Im}\left(\mathbf{m}^{\text {true }}\right)}\right\rangle
\end{aligned}
$$

are thus good indicators of eventual biases which might be introduced by the inversion algorithm. These biases will be displayed in form of vertical bars (see for example Figure 7) and their height should be as close as possible to 0 . We also compute the standard deviations $\mathbf{s}^{\varepsilon_{r}}$ and $\mathbf{s}^{\sigma}$ in each zone, defined separately for the real and imaginary part of $\mathbf{m}^{(*)}$,

$$
\mathbf{s}^{\varepsilon_{r}}=\left\langle\left[\frac{\operatorname{Re}\left(\mathbf{m}^{(*)}-\left\langle\mathbf{m}^{(*)}\right\rangle\right)}{\operatorname{Re}\left(\mathbf{m}^{\text {true }}\right)}\right]^{2}\right\rangle^{1 / 2} \text { and } \mathbf{s}^{\sigma}=\left\langle\left[\frac{\operatorname{Im}\left(\mathbf{m}^{(*)}-\left\langle\mathbf{m}^{(*)}\right\rangle\right)}{\operatorname{Im}\left(\mathbf{m}^{\text {true }}\right)}\right]^{2}\right\rangle^{1 / 2}
$$

These standard deviations are a good indicator of the spreading of the reconstructed values with respect to the expected values. These standard deviations are reported thanks to error bars, which should be as small as possible.

\subsection{Signal-to-noise ratio influence}

In Figure 5, one can see the evolution of the cost functional for various Signal-to-Noise Ratio (SNR) values. The first conclusion that can be drawn is that whatever the values of $\mathrm{SNR}_{b}$ and $\mathrm{SNR}_{m}$, the inversion algorithm behaves in a correct way as it always minimize the cost functional. In very few iterations, there is also a significant reduction (of several $\mathrm{dB}$ ) of the discrepancy between the measured and simulated magnetic field.

The further the initial guess $\mathbf{m}^{(0)}$ is from the true solution $\mathbf{m}^{\text {true }}$ (namely, the smaller $\mathrm{SNR}_{m}$ is), the higher the cost functional stays, even at the end of the minimisation process. It means that, as expected, it is always more efficient to start with initial values which are close to the realistic ones.

The higher the noise level is (namely, the smaller $\mathrm{SNR}_{B}$ is), the higher the cost functional stays. In particular, the final values of $\mathcal{F}$ directly depend on $\mathrm{SNR}_{B}$. As

shown in Figure 5, the offsets between the final $\mathcal{F}$ values are directly proportional to the offsets between the considered $\mathrm{SNR}_{B}$. The value of $\mathrm{SNR}_{B}$ thus provides a minimal threshold that the cost functional $\mathcal{F}$ cannot overcome. 
Figure 7 shows the average biases and standard deviations within each zone for various SNR values. There is no bias for the eye zone as the characteristics of this zone are assumed to be already known. One can see that whatever the SNR values and whatever the zone, the biases $\mathbf{b}^{\varepsilon_{r}}$ and $\mathbf{b}^{\sigma}$ are quite small (less than $10 \%$ in relative error) even if they slightly increase when the initial guess is far from the true solution (for small $\mathrm{SNR}_{m}$ values). This is a clear indication of the robustness of the proposed algorithm.

The biases do not seem to be really affected by an increase of the measurement noise level, that is, a decrease of $\mathrm{SNR}_{B}$. On the contrary, the standard deviations $\mathbf{s}^{\varepsilon_{r}}$ and $\mathbf{s}^{\sigma}$ increase when $\mathrm{SNR}_{B}$ decreases. This behaviour is normal and expected as the errors in the measurement are directly translated into spatial fluctuations of the reconstructed dielectric parameters. If the inverse problem was an over-determined linear problem, it would even be possible to provide a proportional relationship between $\mathbf{s}^{\varepsilon_{r}}, \mathbf{s}^{\sigma}$ and $\mathrm{SNR}_{B}$. In any case, the observed levels of $\mathbf{s}^{\varepsilon_{r}}$ and $\mathbf{s}^{\sigma}$ are rather low and give good confidence in the relevance of this type of approach for MREPT applications.

\subsection{Initial guess influence}

As the inverse problem is an under-determined non-linear problem, there is no guarantee that the minimization algorithm cannot be trapped in local minima. In order to investigate the influence of the initial guess, we have performed 100 runs where the initial guess has been selected randomly according to (30) but for fixed values of $\mathrm{SNR}_{m}$ and $\mathrm{SNR}_{B}$. The values of $\mathrm{SNR}_{m}$ and $\mathrm{SNR}_{B}$ have been selected in order to positioned ourselves in an plausible scenario, given the SNRs provided in [40]. We have then

gathered these 100 final reconstructions values $\mathbf{m}^{(*)}$ and computed their average over these 100 runs. Figure 8 shows the resulting biases and standard-deviations for each zone.

Whatever the zone, the algorithm always find the correct values as there are nearly no biases (less than $1 \%$ in relative error). There is also nearly no spreading in the final biases and standard deviations. It means that, in the considered configuration, the algorithm always converges towards the same local minimum which corresponds to the true solution. One reason for this nice behaviour could be explained by the fact that the virtual receivers are located directly inside the area for which we are searching the dielectric parameters. Compared to classical microwave imaging setups where the receivers are outside of the area to characterize, the inverse problem might be here better conditioned.

\subsection{Transmitters influence}

Figure 9 shows the evolution of the cost functional when the number of transmitters $N_{s}$ increases. As the cost functional is weighted according to $N_{s}$ and as $\mathrm{SNR}_{B}$ is constant, the final $\mathcal{F}$ values are the same whatever the values of $N_{s}$. As compared to Figure 5 
where $N_{s}=1$, one can directly see that an increase in the number of transmitters leads to a faster convergence rate.

Figure 10 shows the final biases and standard deviations for several $N_{s}$. It seems that changing $N_{s}$ has little effect on the systematic errors. This is in accordance with the previous comments regarding the behaviour of the biases, which appear unaffected by the parameters choices. When $N_{s}$ increases, the standard deviations decrease, as one could expect. One might have also hoped for a direct relationship between $N_{s}$ and s. Unfortunately, doubling $N_{s}$ does not reduce by half the standard deviations, it only decreases them as the inverse problem is non-linear.

\subsection{Regularisation influence}

Finally, we investigate the effects of incorporating a-priori information. The cost functionals associated to the element-based inversion and the zone-based inversion can be seen in Figure 11. Definitely, as the problem is over-determined, it requires very few iterations when the geometrical priors are used. Figure 12 shows the final reconstructed map $\mathbf{m}^{(*)}$ which is nearly identical to the one shown in Figure 3. A quantitative appraisal of the difference between these two maps has shown that there were no biases in the reconstructed maps. As expected, $\mathbf{s}^{\varepsilon_{r}}=\mathbf{s}^{\sigma}=0$ as we have assumed the permittivity and conductivity to be constant in each zone. Another interesting point is that the biases are smaller than in the element-based inversion, even at very low $\mathrm{SNR}_{m}$.

Of course, the segmentation algorithm might have an effect on the zone-based inversion results but one should recall that the spatial resolution of high-field MRI images is in the millimetre range while a classical RF microwave imaging system has a spatial resolution close to $\lambda / 2$, where the wavelength $\lambda$ in the brain is in the decimetre range at the Larmor frequency of $298 \mathrm{MHz}$.

\section{Conclusion}

In this paper, we have investigated a MREPT gradient-based inversion algorithm to quantitatively recover the permittivity values of brain tissues based on $B_{1}^{+}$mapping, which can be acquired in MRI setups. Such an inversion has been performed with an electric field formulation and an ad-hoc adjoint field derivation. The possibility of incorporating geometrical priors has also been offered.

The numerical tests have shown the robustness and the correct behaviour of the proposed algorithm, even at low SNR. As expected, adding more transmitters or increasing the SNR reduces the standard deviations of the reconstructed maps. As well, the use of a priori information leads to faster convergence rate and smaller estimation biases. Moreover, the algorithm does not seem to converge towards unwanted local minima.

It would interesting to investigate the implementation of such an inversion scheme considering a full three-dimensional configuration and of course, to confront it to 
experimental dataset. As well, it would be worth comparing the results that could be obtained with $\left|B_{1}^{+}\right|$mapping with respect to the ones obtained here with an amplitude and phase $B_{1}^{+}$mapping. This would require some modifications in the cost functional but mainly in the adjoint field expression [41]. Finally, it would be interesting to compare the degree of non-linearity and ill-posedness of this MREPT problem with the one of a classical microwave imaging system, where the electromagnetic field can only be measured out of the human body.

\section{Acknowledgments}

A. Rahimov would like to acknowledge the support of the Azerbaijan National Academy of Science during his stay in France. A. Litman wishes to acknowledge the support of COST Action TD1301, MiMed. The authors are also very grateful to H. Tortel for fruitful discussions, in particular for his help in the forward problem formulation. The authors would also like to thank M. Luong and A. France for exchanging on this specific MRI problem.

\section{Appendix A. Electromagnetic equations}

According to Maxwell's equations, under the $\exp (-j \omega t)$ time convention, the electromagnetic fields satisfy

$$
\nabla \times \vec{B}=-j \omega \varepsilon_{r}(\vec{r}) \varepsilon_{0} \mu_{0} \vec{E}+\mu_{0} \vec{J} \text { and } \nabla \times \vec{E}=j \omega \vec{B}
$$

where $\mu_{0}$ is the vacuum magnetic permeability as all the materials are non-magnetic. The current density $\vec{J}$ can be decomposed as $\vec{J}=\vec{J}_{\text {ohm }}+\vec{J}_{\text {source, }}$, where $\vec{J}_{\text {ohm }}=\sigma \vec{E}$. Thus

$$
\begin{aligned}
\nabla \times \vec{B} & =-j \omega \varepsilon_{r}(\vec{r}) \varepsilon_{0} \mu_{0} \vec{E}+\mu_{0} \sigma \vec{E}+\mu_{0} \vec{J}_{\text {source }} \\
& =-j \omega \varepsilon_{0} \mu_{0} \underbrace{\left(\varepsilon_{r}(\vec{r})+j \frac{\sigma(\vec{r})}{\omega \varepsilon_{0}}\right)}_{m(\vec{r})} \vec{E}+\mu_{0} \vec{J}_{\text {source }} \\
& =-\frac{j}{\omega}\left[\omega^{2} \varepsilon_{0} \mu_{0} m(\vec{r}) \vec{E}+j \mu_{0} \omega \vec{J}_{\text {source }}\right]=-\frac{j}{\omega}\left[k^{2} m(\vec{r}) \vec{E}-\vec{S}\right]
\end{aligned}
$$

This corresponds to (3), where $k=\omega \sqrt{\varepsilon_{0} \mu_{0}}$ is the wave number in air and the excitation source term is defined as $\vec{S}=-j \mu_{0} \omega \vec{J}_{\text {source }}$.

\section{Appendix B. Gâteaux derivative for the fields}

\section{Appendix B.1. Derivation with respect to the Lagrange multipliers}

We apply the definition of the Gâteaux derivative with respect to $U\left(\cdot ; \vec{r}_{s}\right)$ to the Lagrangian functional (14), that is,

$\lim _{t \rightarrow 0} \frac{\mathcal{L}(E, U+t v, m)-\mathcal{L}(E, U, m)}{t}$ 
MRI-Based Electric Properties Tomography with a Quasi-Newton approach

$$
\begin{aligned}
& =\lim _{t \rightarrow 0} \sum_{s=1}^{N_{s}} \frac{\mathcal{L}_{s}(E, U+t v, m)-\mathcal{L}_{s}(E, U, m)}{t} \\
& =\lim _{t \rightarrow 0} \frac{\mathcal{L}_{s}(E, U+t v, m)-\mathcal{L}_{s}(E, U, m)}{t} \quad \text { (using (15)) } \\
& =\lim _{t \rightarrow 0} \frac{1}{t} \operatorname{Re}\left[\mathcal{H}_{s}\left(E\left(\cdot ; \vec{r}_{s}\right), U\left(\cdot ; \vec{r}_{s}\right)+t v, m\right)-\mathcal{H}_{s}\left(E\left(\cdot ; \vec{r}_{s}\right), U\left(\cdot ; \vec{r}_{s}\right), m\right)\right] \\
& =\lim _{t \rightarrow 0} \frac{1}{t} \operatorname{Re}\left[\left\langle\Delta E+k^{2} m E-S\left(\cdot ; \vec{r}_{s}\right) \mid U\left(\cdot ; \vec{r}_{s}\right)+t v\right\rangle_{\Omega} \quad\right. \text { (using (16)) } \\
& \qquad \lim _{t \rightarrow 0} \operatorname{Re} \frac{\left\langle\Delta E+k^{2} m E-S\left(\cdot ; \vec{r}_{s}\right) \mid t v\right\rangle_{\Omega}}{t}=\operatorname{Re}\left\langle\Delta E+k^{2} m E-S\left(\cdot ; \vec{r}_{s}\right) \mid v\right\rangle_{\Omega} .
\end{aligned}
$$

In that case, we end up with a Fréchet derivative for the Lagrange multipliers (19).

Appendix B.2. Derivation with respect to the electrical field

We apply the definition of the Gâteaux derivative with respect to $E\left(\cdot ; \vec{r}_{s}\right)$ to the Lagrangian functional (14), that is,

$$
\begin{aligned}
& \lim _{t \rightarrow 0} \frac{\mathcal{L}(E+t v, U, m)-\mathcal{L}(E, U, m)}{t} \\
&=\lim _{t \rightarrow 0} \sum_{s=1}^{N_{s}} \frac{\mathcal{L}_{s}(E+t v, U, m)-\mathcal{L}_{s}(E, U, m)}{t} \\
&=\lim _{t \rightarrow 0} \frac{\mathcal{L}_{s}(E+t v, U, m)-\mathcal{L}_{s}(E, U, m)}{t} \\
&=\lim _{t \rightarrow 0} \frac{1}{t}\left[\mathcal{F}_{s}\left(E\left(\cdot ; \vec{r}_{s}\right)+t v\right)-\mathcal{F}_{s}\left(E\left(\cdot ; \vec{r}_{s}\right)\right)\right] \\
&+\lim _{t \rightarrow 0} \frac{1}{t} \operatorname{Re}\left[\mathcal{H}_{s}\left(E\left(\cdot ; \vec{r}_{s}\right)+t v, U\left(\cdot ; \vec{r}_{s}\right), m\right)-\mathcal{H}_{s}\left(E\left(\cdot ; \vec{r}_{s}\right), U\left(\cdot ; \vec{r}_{s}\right), m\right)\right] \\
&= \lim _{t \rightarrow 0} \frac{1}{t}\left[\mathcal{F}_{s}\left(E\left(\cdot ; \vec{r}_{s}\right)+t v\right)-\mathcal{F}_{s}\left(E\left(\cdot ; \vec{r}_{s}\right)\right)\right]+\operatorname{Re}\left\langle\Delta v+k^{2} m v \mid U\right\rangle_{\Omega}
\end{aligned}
$$

thanks to the definition of $\mathcal{H}_{s}$ in (16). Let us now focus on the first term in brackets in the previous equation

$$
\begin{aligned}
\lim _{t \rightarrow 0} \frac{1}{t}\left[\mathcal{F}_{s}\left(E\left(\cdot ; \vec{r}_{s}\right)+t v\right)-\mathcal{F}_{s}\left(E\left(\cdot ; \vec{r}_{s}\right)\right)\right] \\
=\lim _{t \rightarrow 0} \frac{1}{t} \frac{\omega^{2}}{2 N_{s}} \sum_{r=1}^{N_{r}} \int_{\Omega}\left|B_{1}^{+}(E+t v)\left(\vec{r} ; \vec{r}_{s}\right)-B_{1}^{+, \text {meas }}\left(\vec{r} ; \vec{r}_{s}\right)\right|^{2} \delta\left(\vec{r}-\vec{r}_{r}\right) d \vec{r} \\
\quad-\lim _{t \rightarrow 0} \frac{1}{t} \frac{\omega^{2}}{2 N_{s}} \sum_{r=1}^{N_{r}} \int_{\Omega}\left|B_{1}^{+}(E)\left(\vec{r} ; \vec{r}_{s}\right)-B_{1}^{+, \text {meas }}\left(\vec{r} ; \vec{r}_{s}\right)\right|^{2} \delta\left(\vec{r}-\vec{r}_{r}\right) d \vec{r} .
\end{aligned}
$$

As $B_{1}^{+}$is a linear operator with respect to $E$ (see (8)), we have

$$
\begin{aligned}
& \left|B_{1}^{+}(E+t v)-B_{1}^{+, \text {meas }}\right|^{2}=\left|B_{1}^{+}(E)-B_{1}^{+, \text {meas }}+t B_{1}^{+}(v)\right|^{2} \\
& =\left|B_{1}^{+}(E)-B_{1}^{+, \text {meas }}\right|^{2}+t^{2}\left|B_{1}^{+}(v)\right|^{2} \\
& +2 t \operatorname{Re}\left(\overline{\left[B_{1}^{+}(E)-B_{1}^{+, \text {meas }}\right]} B_{1}^{+}(v)\right) \text {. }
\end{aligned}
$$


Replacing (B.4) into (B.3), we obtain

$$
\begin{aligned}
\lim _{t \rightarrow 0} \frac{1}{t}\left[\mathcal{F}_{s}\left(E\left(\cdot ; \vec{r}_{s}\right)+t v\right)-\mathcal{F}_{s}\left(E\left(\cdot ; \vec{r}_{s}\right)\right)\right] \\
=\lim _{t \rightarrow 0} \frac{1}{t} \frac{\omega^{2}}{2 N_{s}} \sum_{r=1}^{N_{r}} \int_{\Omega}\left\{t^{2}\left|B_{1}^{+}(v)\right|^{2}\right. \\
\left.\quad+2 t \operatorname{Re}\left(\overline{\left[B_{1}^{+}(E)\left(\vec{r} ; \vec{r}_{s}\right)-B_{1}^{+, \text {meas }}\left(\vec{r} ; \vec{r}_{s}\right)\right]} B_{1}^{+}(v)\right)\right\} \delta\left(\vec{r}-\vec{r}_{r}\right) d \vec{r} \\
=\frac{\omega^{2}}{N_{s}} \operatorname{Re} \sum_{r=1}^{N_{r}} \int_{\Omega} \frac{\left[B_{1}^{+}(E)\left(\vec{r} ; \vec{r}_{s}\right)-B_{1}^{+, \text {meas }}\left(\vec{r} ; \vec{r}_{s}\right)\right]}{B_{1}^{+}(v) \delta\left(\vec{r}-\vec{r}_{r}\right) d \vec{r}}
\end{aligned}
$$

Finally, by substituing (B.5) into (B.2), we get (20), that is,

$$
\begin{aligned}
& \lim _{t \rightarrow 0} \frac{\mathcal{L}(E+t v, U, m)-\mathcal{L}(E, U, m)}{t}=\operatorname{Re}\left\langle\Delta v+k^{2} m v \mid U\left(\cdot ; \vec{r}_{s}\right)\right\rangle_{\Omega} \\
& \quad+\frac{\omega^{2}}{N_{s}} \operatorname{Re} \sum_{r=1}^{N_{r}} \int_{\Omega} \overline{\left[B_{1}^{+}(E)\left(\vec{r} ; \vec{r}_{s}\right)-B_{1}^{+, \text {meas }}\left(\vec{r} ; \vec{r}_{s}\right)\right]} B_{1}^{+}(v) \delta\left(\vec{r}-\vec{r}_{r}\right) d \vec{r} .
\end{aligned}
$$

Unfortunately $B_{1}^{+}$can not be further decomposed and the previous derivative can not fully written in terms of a Fréchet derivative. This is nevertheless not an issue, as it is still possible to impose a saddle-point condition based on such expression. Indeed, (17) implies that

$$
\begin{aligned}
\left\langle\Delta v+k^{2} m v\right| & \left.U\left(\cdot ; \vec{r}_{s}\right)\right\rangle_{\Omega} \\
= & -\frac{\omega^{2}}{N_{s}} \sum_{r=1}^{N_{r}} \int_{\Omega} \overline{\left[B_{1}^{+}(E)\left(\vec{r} ; \vec{r}_{s}\right)-B_{1}^{+, \text {meas }}\left(\vec{r} ; \vec{r}_{s}\right)\right]} B_{1}^{+}(v) \delta\left(\vec{r}-\vec{r}_{r}\right) d \vec{r} .
\end{aligned}
$$

Applying the first Green formula, we transform the left side of (B.6) as follows

$$
\begin{aligned}
\left\langle\Delta v+k^{2} m v \mid U\left(\cdot ; \vec{r}_{s}\right)\right\rangle_{\Omega} & =\int_{\Omega}\left(\Delta v+k^{2} m v\right) \bar{U}\left(\cdot ; \vec{r}_{s}\right) d \vec{r} \\
& =-\int_{\Omega} \nabla v \nabla \bar{U}\left(\cdot ; \vec{r}_{s}\right) d \vec{r}+\int_{\partial \Omega} \frac{\partial v}{\partial n} \bar{U}\left(\vec{r} ; \vec{r}_{s}\right) d s+\int_{\Omega} k^{2} m \bar{U}\left(\cdot ; \vec{r}_{s}\right) v d \vec{r} \\
& =\int_{\Omega}\left(-\nabla v \nabla \bar{U}\left(\cdot ; \vec{r}_{s}\right)+k^{2} m v \bar{U}\left(\vec{r} ; \vec{r}_{s}\right)\right) d \vec{r} .
\end{aligned}
$$

if $U$ is assumed to be null on the boundary of the computational domain. Taking into account the last expression in (B.6) and by noting $P\left(\cdot ; \vec{r}_{s}\right)=\bar{U}\left(\cdot ; \vec{r}_{s}\right)$, we obtain $(21)$, that is,

$$
\begin{aligned}
\int_{\Omega}\left(-\nabla v \nabla P\left(\cdot ; \vec{r}_{s}\right)+k^{2} m v P\left(\cdot ; \vec{r}_{s}\right)\right) d \vec{r} \\
\quad=-\frac{\omega^{2}}{N_{s}} \sum_{r=1}^{N_{r}} \int_{\Omega} \overline{\left[B_{1}^{+}(E)\left(\vec{r} ; \vec{r}_{s}\right)-B_{1}^{+, \text {meas }}\left(\vec{r} ; \vec{r}_{s}\right)\right]} B_{1}^{+}(v) \delta\left(\vec{r}-\vec{r}_{r}\right) d \vec{r} .
\end{aligned}
$$




\section{Appendix C. Fréchet derivative for the complex permittivity $m$}

As done previously in Appendix B, we get

$$
\begin{aligned}
& \lim _{t \rightarrow 0} \frac{\mathcal{L}(E, U, m+t v)-\mathcal{L}(E, U, m)}{t}=\lim _{t \rightarrow 0} \sum_{s=1}^{N_{s}} \frac{\mathcal{L}_{s}(E, U, m+t v)-\mathcal{L}_{s}(E, U, m)}{t} \\
& =\lim _{t \rightarrow 0} \frac{1}{t} \operatorname{Re} \sum_{s=1}^{N_{s}}\left[\mathcal{H}_{s}\left(E\left(\cdot ; \vec{r}_{s}\right), U\left(\cdot ; \vec{r}_{s}\right), m+t v\right)-\mathcal{H}_{s}\left(E\left(\cdot ; \vec{r}_{s}\right), U\left(\cdot ; \vec{r}_{s}\right), m\right)\right] \\
& =\lim _{t \rightarrow 0} \frac{1}{t} \operatorname{Re} \sum_{s=1}^{N_{s}}\left[\left\langle\Delta E+k^{2}(m+t v) E-S\left(\cdot ; \vec{r}_{s}\right) \mid U\left(\cdot ; \vec{r}_{s}\right)\right\rangle_{\Omega}-\left\langle\Delta E+k^{2} m E-S\left(\cdot ; \vec{r}_{s}\right) \mid U\left(\cdot ; \vec{r}_{s}\right)\right\rangle_{\Omega}\right] \\
& =\lim _{t \rightarrow 0} \frac{1}{t}\left[t \operatorname{Re} \sum_{s=1}^{N_{s}}\left\langle k^{2} v E \mid U\left(\cdot ; \vec{r}_{s}\right)\right\rangle_{\Omega}\right]=\operatorname{Re} \sum_{s=1}^{N_{s}}\left\langle k^{2} v E\left(\cdot ; \vec{r}_{s}\right) \mid U\left(\cdot ; \vec{r}_{s}\right)\right\rangle_{\Omega} \\
& =\operatorname{Re} \sum_{s=1}^{N_{s}}\left\langle v \mid k^{2} \bar{E}\left(\cdot ; \vec{r}_{s}\right) U\left(\cdot ; \vec{r}_{s}\right)\right\rangle_{\Omega}=\operatorname{Re} \sum_{s=1}^{N_{s}}\left\langle k^{2} \bar{E}\left(\cdot ; \vec{r}_{s}\right) U\left(\cdot ; \vec{r}_{s}\right) \mid v\right\rangle_{\Omega} \\
& =\operatorname{Re} \sum_{s=1}^{N_{s}}\left\langle k^{2} \bar{E}\left(\cdot ; \vec{r}_{s}\right) \bar{P}\left(\cdot ; \vec{r}_{s}\right) \mid v\right\rangle_{\Omega}=\operatorname{Re}\left\langle k^{2} \sum_{s=1}^{N_{s}} \bar{E}\left(\cdot ; \vec{r}_{s}\right) \bar{P}\left(\cdot ; \vec{r}_{s}\right) \mid v\right\rangle_{\Omega} .
\end{aligned}
$$

as $P=\bar{U}$ and $\operatorname{Re}\langle u \mid v\rangle_{\Omega}=\operatorname{Re}\langle v \mid u\rangle_{\Omega}$. Using the definition of complex derivatives of real-valued functions $[35,36]$, from the last expression, we obtain the gradient of the cost functional (23).

\section{References}

[1] U. Katscher, D.H. Kim, and J.K. Seo. Recent Progress and Future Challenges in MR Electric Properties Tomography. Computational and Mathematical Methods in Medicine, 2013:546562, 2013.

[2] J.K. Seo, E.J. Woo, U. Katscher, and Y. Wang. Electro-Magnetic Tissue Properties MRI. Imperial College Press, London, 2014.

[3] X. Zhang, J. Liu, and B. He. Magnetic-resonance-based electrical properties tomography: A review. IEEE Review Biomed. Eng., 7:87-96, 2014.

[4] M. Lazebnik, D. Popovic, L. McCartney, C.B. B Watkins, M.J. Lindstrom, J. Harter, S. Sewall, T. Ogilvie, A. Magliocco, T.M. Breslin, W. Temple, D. Mew, J.H. Booske, M. Okoniewski, and S.C. Hagness. A large-scale study of the ultrawideband microwave dielectric properties of normal, benign and malignant breast tissues obtained from cancer surgeries. Physics in Medicine and Biology, 52(21):6093, 2007.

[5] R. C. Conceicao, J.J. Mohr, and M. O'Halloran, editors. An introduction to microwave imaging for breast cancer detection. Biological and Medical Physics, Biomedical Engineering. Springer, Switzerland, 2016.

[6] M. de Greef, O. Ipek, A.J.E. Raaijmakers, J. Crezee, and C.A.T. van den Berg. Specific Absorption Rate Intersubject Variability in 7T Parallel Transmit MRI of the Head. Magnetic Resonance in Medecine, 69(5):1476-1485, May 2013.

[7] E.M. Haacke, L.S. Petropoulos, E.W. Nilges, and D.H. Wu. Extraction of conductivity and permittivity using magnetic resonance imaging. Physics in Medicine and Biology, 38(6):471-477, 1991. 
[8] S. Akoka, F. Franconi, F. Seguin, and A. Le Pape. Radiofrequency map of an NMR coil by imaging. Magnetic Resonance Imaging, 11(3):437-441, 1993.

[9] D.I. Hoult. The principle of reciprocity in signal strength calculations - A mathematical guide. Concepts in Magnetic Resonance, 12(4):173-187, 2000.

[10] R W. Brown, Y.-C.N. Cheng, E.M. Haacke, M.R. Thompson, and R. Venkatesan. Magnetic Resonance Imaging: Physical Principles and Sequence Design. Wiley-Blackwell, Hoboken, New Jersey, 2nd edition, 2014.

[11] V.L. Yarnykh. Actual flip-angle imaging in the pulsed steady state: a method for rapid threedimensional mapping of the transmitted radiofrequency field. Magnetic Resonance in Medecine, 57(1):192-200, 2007.

[12] G.R. Morrell. A phase-sensitive method of flip angle mapping. Magnetic Resonance in Medicine, 60(4):889-894, 2008.

[13] L.I. Sacolick, F. Wiesinger, I. Hancu, and M.W. Vogel. B1 mapping by Bloch-Siegert shift. Magnetic Resonance in Medicine, 63(5):1315-1322, 2010.

[14] N. Choi, J. Lee, M.-O. Kim, J. Shin, and D.-H. Kim. A modified multi-echo AFI for simultaneous $b_{1}^{+}$magnitude and phase mapping. Magnetic Resonance Imaging, 32(4):314 - 320, 2014.

[15] A.L.H.M.W. van Lier, D.O. Brunner, K.P. Pruessmann, D.W.J. Klomp, P.R. Luijten, J.J.W. Lagendijk, and C.A.T. van den Berg. $b_{1}^{+}$phase mapping at $7 \mathrm{~T}$ and its application for in vivo electrical conductivity mapping. Magnetic Resonance in Medicine, 67(2):552-561, 2012.

[16] U. Katscher, T. Voigt, C. Findeklee, P. Vernickel, K. Nehrke, and O. Doessel. Determination of electric conductivity and local SAR via B1 mapping. IEEE Trans. Med. Imag., 28(9):1365-1374, 2009.

[17] T. Voigt, U. Katscher, and O. Doessel. Quantitative conductivity and permittivity imaging of the human brain using electric properties tomography. Magnetic Resonance in Medecine, 66(2):456466, 2011.

[18] J.K. Seo, D.H. Kim, J. Lee, O.I. Kwon, S.Z.K. Sajub, and E.J. Woo. Electrical tissue property imaging using MRI at DC and Larmor frequency. Inverse Problems, 28:084002, 2012.

[19] A. Borsic, I. Perreard, A. Mahara, and R. J. Halter. An inverse problems approach to MR-EPT image reconstruction. IEEE Trans. Med. Imag., 35(1):244-256, Jan 2016.

[20] A.I. Nachman, D. Wang, W. Ma, and M.L. Joy. A local formula for inhomogeneous complex conductivity as a function of the RF magnetic field. In Proc. 15th Annual Meeting of ISMRM, 2013.

[21] F.S. Hafalir, O.F. Oran, N. Gurler, and Y.Z. Ider. Convection-reaction equation based magnetic resonance electrical properties tomography (cr-MREPT). IEEE Trans. Med. Imag., 33(3):777793, 2014.

[22] H. Ammari, H. Kwon, Y. Lee, K. Kang, and J.K. Seo. Magnetic resonance-based reconstruction method of conductivity and permittivity distributions at the Larmor frequency. Inverse Problems, 31(10):105001, 2015.

[23] E. Balidemaj, C.A.T. van den Berg, J. Trinks, A.L.H.M.W. van Lier, A.J. Nederveen, L.J.A. Stalpers, H. Crezee, and R.F. Remis. CSI-EPT: A contrast source inversion approach for improved MRI-based electric properties tomography. IEEE Trans. Med. Imag., 34(9):17881796, Sept 2015.

[24] A. Deistung, F. Schweser, and J.R. Reichenbach. Overview of quantitative susceptibility mapping. NMR in Biomedicine, 30(4):e3569, 2017.

[25] J. Nocedal and S.J. Wright. Numerical Optimization. Springer Series in Operations Research. Springer, New-York, 2006.

[26] D. Bertsekas. Constrained Optimization and Lagrange Multiplier Methods. Academic Press, 1982.

[27] I. Voznyuk, A. Litman, and H. Tortel. Efficient combination of a 3D Quasi-Newton inversion algorithm and a vector dual-primal finite element tearing and interconnecting method. Inverse Problems, 31:085005, 2015.

[28] C. Gabriel, S. Gabriel, and E. Corthout. The dielectric properties of biological tissues: I. literature 
survey. Physics in Medicine and Biology, 41(11):2231, 1996.

[29] B. van den Bergen, C.C. Stolk, J.B. van den Berg, J.J.W Lagendijk, and C.A.T. Van den Berg. Ultra fast electromagnetic field computations for RF multi-transmit techniques in high field MRI. Physics in Medicine and Biology, 54(5):1253, 2009.

[30] R.F. Lee, C.R. Westgate, R.G. Weiss, D.C. Newman, and P.A. Bottomley. Planar strip array (PSA) for MRI. Magnetic Resonance in Medicine, 45(4):673-683, 2001.

[31] J.-M. Jin. The Finite Element Method in Electromagnetics. Wiley-IEEE Press, New York, 2nd edition, 2002.

[32] I. Voznyuk, H. Tortel, and A. Litman. Scattered field computation with an extended FETI-DPEM2 method. Progress In Electromagnetics Research, 139:247-263, 2013.

[33] C. Geuzaine and J.F. Remacle. GMSH: A three dimensional finite element mesh generator with built-in pre- and post-processing facilities. International Journal for Numerical Methods in Engineering, 79(11):1309-1331, 2009. See internet address: http://www.geuz.org/gmsh/.

[34] P. R. Amestoy, I. S. Duff, and J. Y. L'Excellent. Multifrontal parallel distributed symmetric and unsymmetric solvers. Computer Methods in Applied Mechanics and Engineering, 184(24):501$520,2000$.

[35] R. Remmert. Theory of Complex Functions. Springer-Verlag, New-York, 1991.

[36] K. Kreutz-Delgado. The Complex Gradient Operator and the CR-Calculus, 2009. arXiV:0906.4835.

[37] R. Byrd, P. Lu, J. Nocedal, and C. Zhu. A limited memory algorithm for bound constrained optimization. SIAM Journal on Scientific Computing, 16(5):1190-1208, September 1995.

[38] C. Zhu, R.H. Byrd, P. Lu, and J. Nocedal. Algorithm 778: L-BFGS-B: Fortran subroutines for large-scale bound-constrained optimization. ACM Trans. Math. Softw., 23(4):550-560, December 1997.

[39] G.C Scott, M.L.G Joy, R.L Armstrong, and R.M Henkelman. Sensitivity of magnetic-resonance current-density imaging. Journal of Magnetic Resonance, 97(2):235 - 254, 1992.

[40] R. Pohmann, O. Speck, and K. Scheffler. Signal-to-noise ratio and MR tissue parameters in human brain imaging at 3, 7, and 9.4 tesla using current receive coil arrays. Magnetic Resonance in Medicine, 75(2):801-809, 2016.

[41] A. Litman and K. Belkebir. Two-dimensional inverse profiling problem using phaseless data. $J$. Opt. Soc. Am. A, 23(11):2737-2746, Nov 2006. 


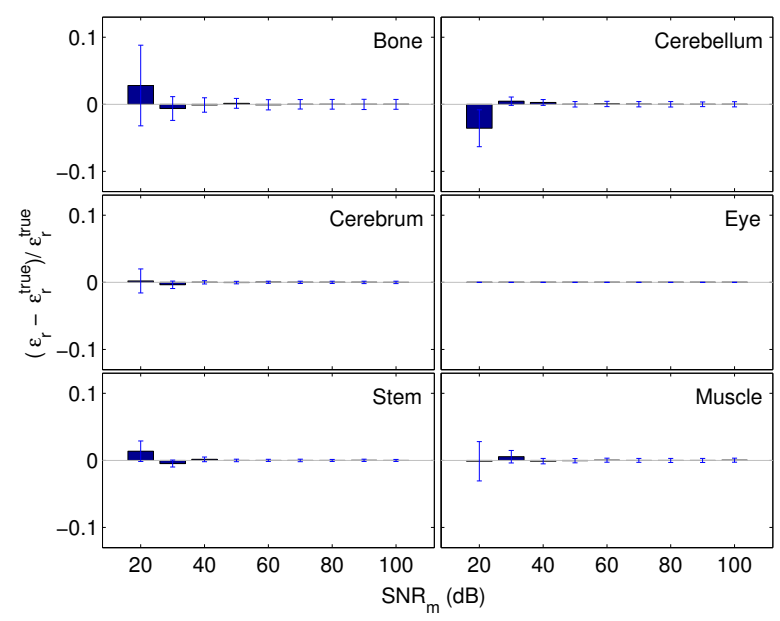

(a) $\mathbf{b}^{\varepsilon_{r}}$ and $\mathbf{s}^{\varepsilon_{r}}, \mathrm{SNR}_{B}=70 \mathrm{~dB}$

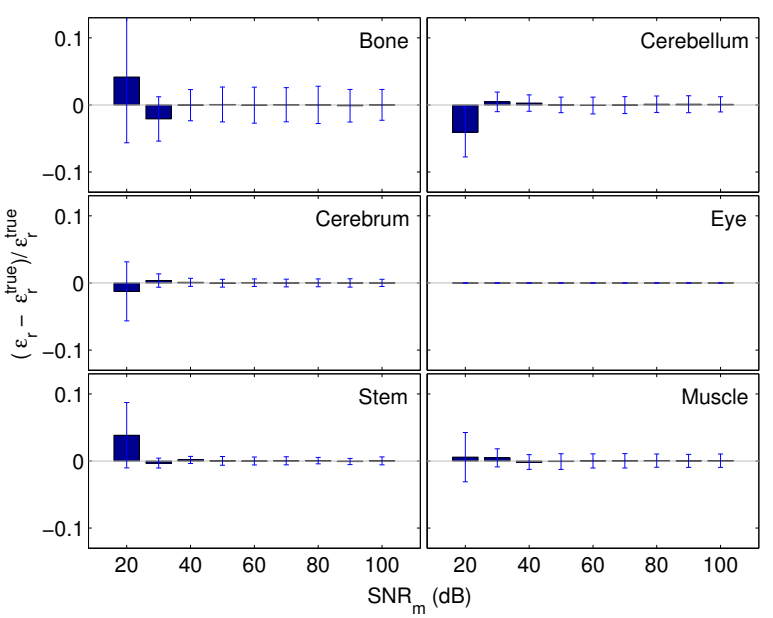

(c) $\mathbf{b}^{\varepsilon_{r}}$ and $\mathbf{s}^{\varepsilon_{r}}, \mathrm{SNR}_{B}=60 \mathrm{~dB}$

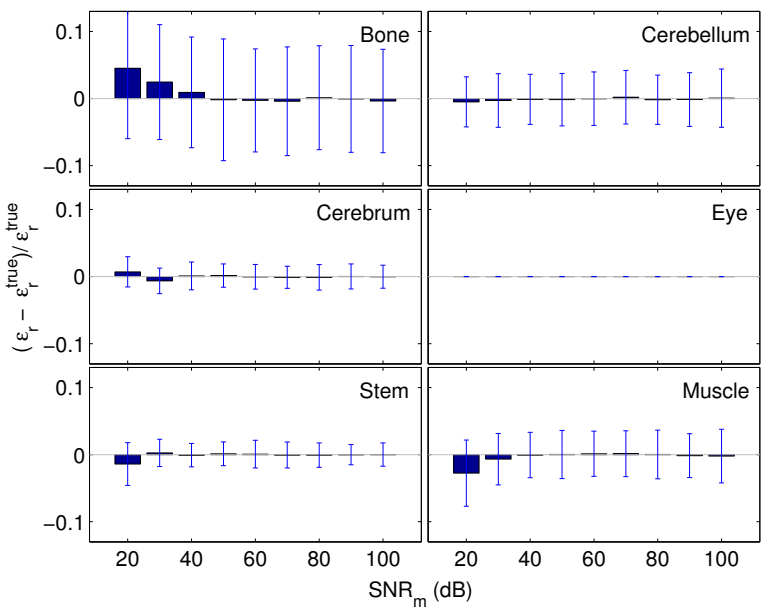

(e) $\mathbf{b}^{\varepsilon_{r}}$ and $\mathbf{s}^{\varepsilon_{r}}, \mathrm{SNR}_{B}=50 \mathrm{~dB}$

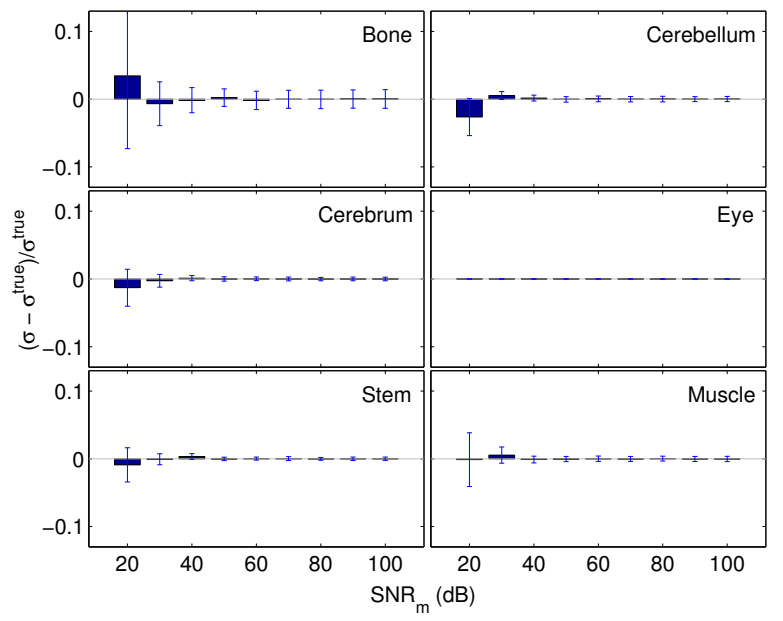

(b) $\mathbf{b}^{\sigma}$ and $\mathbf{s}^{\sigma}, \mathrm{SNR}_{B}=70 \mathrm{~dB}$

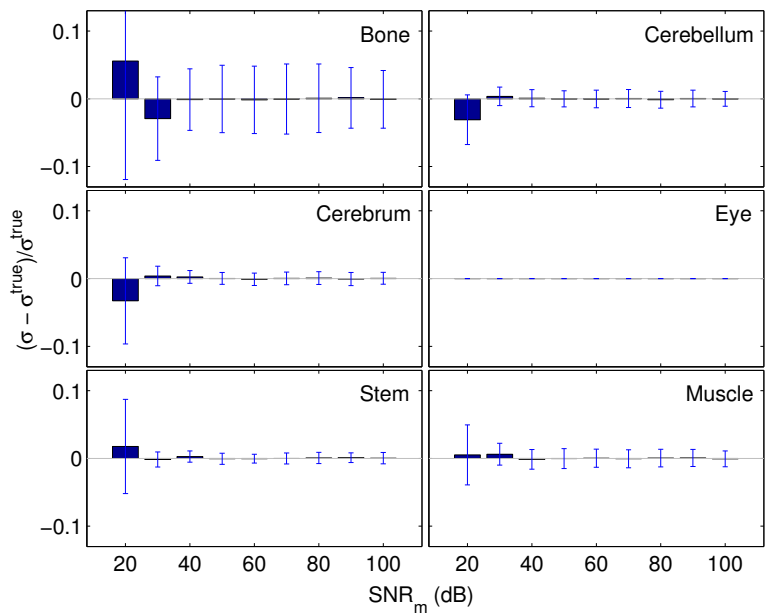

(d) $\mathbf{b}^{\sigma}$ and $\mathbf{s}^{\sigma}, \mathrm{SNR}_{B}=60 \mathrm{~dB}$

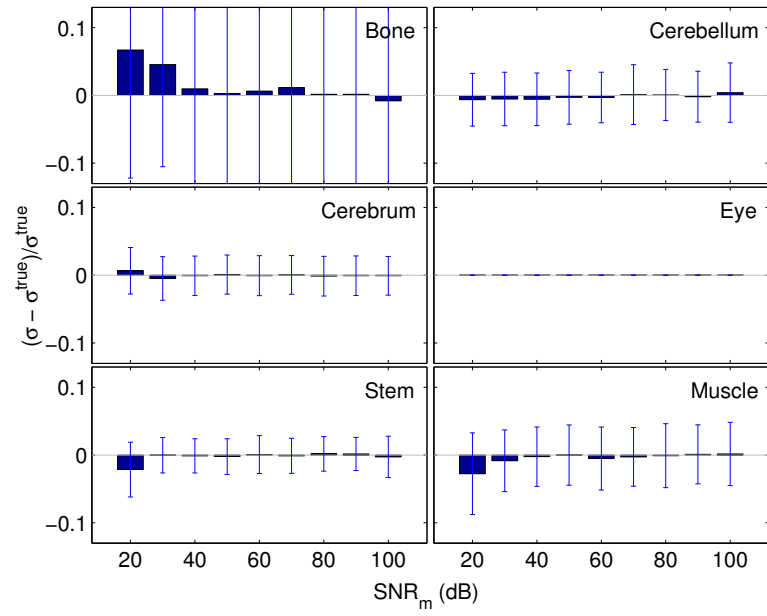

(f) $\mathbf{b}^{\sigma}$ and $\mathbf{s}^{\sigma}, \mathrm{SNR}_{B}=50 \mathrm{~dB}$

Figure 7. Biases $\mathbf{b}^{\varepsilon_{r}}$ and $\mathbf{b}^{\sigma}$ (represented by the vertical bars) combined with standard deviations $\mathbf{s}^{\varepsilon_{r}}$ and $\mathbf{s}^{\sigma}$ (represented by the error bars) for various $\operatorname{SNR}_{m}\left(N_{s}=1\right.$, element-based inversion). 


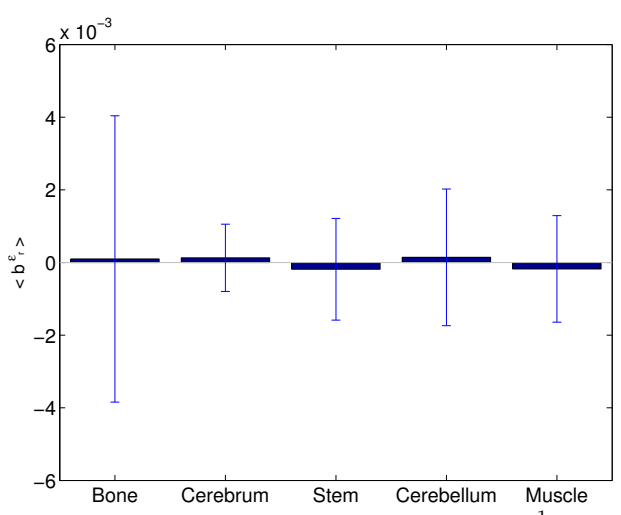

(a) $\left\langle\mathbf{b}^{\varepsilon_{r}}\right\rangle$ and $\left\langle\left(\mathbf{b}^{\varepsilon_{r}}-\left\langle\mathbf{b}^{\varepsilon_{r}}\right\rangle\right)^{2}\right\rangle^{\frac{1}{2}}$

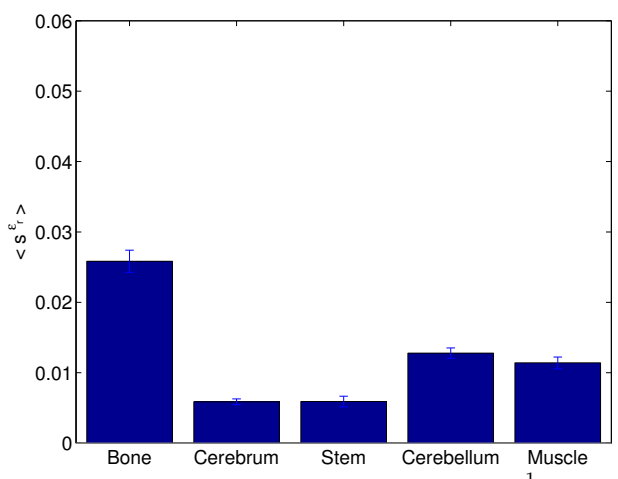

(c) $\left\langle\mathbf{s}^{\varepsilon_{r}}\right\rangle$ and $\left\langle\left(\mathbf{s}^{\varepsilon_{r}}-\left\langle\mathbf{s}^{\varepsilon_{r}}\right\rangle\right)^{2}\right\rangle^{\frac{1}{2}}$

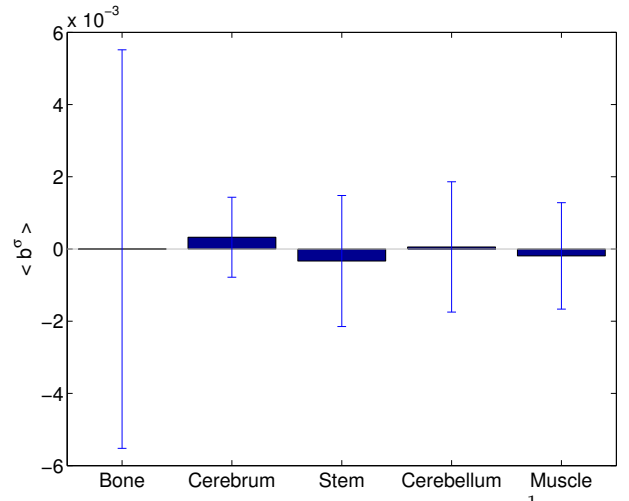

(b) $\left\langle\mathbf{b}^{\sigma}\right\rangle$ and $\left\langle\left(\mathbf{b}^{\sigma}-\left\langle\mathbf{b}^{\sigma}\right\rangle\right)^{2}\right\rangle^{\frac{1}{2}}$

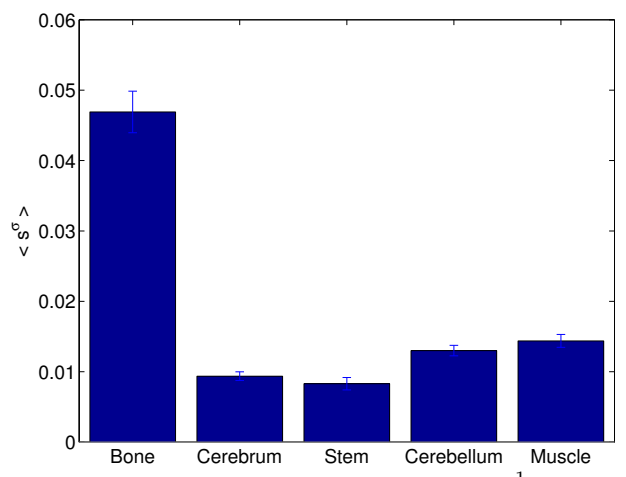

(d) $\left\langle\mathbf{s}^{\sigma}\right\rangle$ and $\left\langle\left(\mathbf{s}^{\sigma}-\left\langle\mathbf{s}^{\sigma}\right\rangle\right)^{2}\right\rangle^{\frac{1}{2}}$

Figure 8. Average over 100 inverse problems runs of the final biases and standard deviations ( $\mathrm{SNR}_{m}=40 \mathrm{~dB}, \mathrm{SNR}_{B}=60 \mathrm{~dB}, N_{s}=1$, element-based inversion).
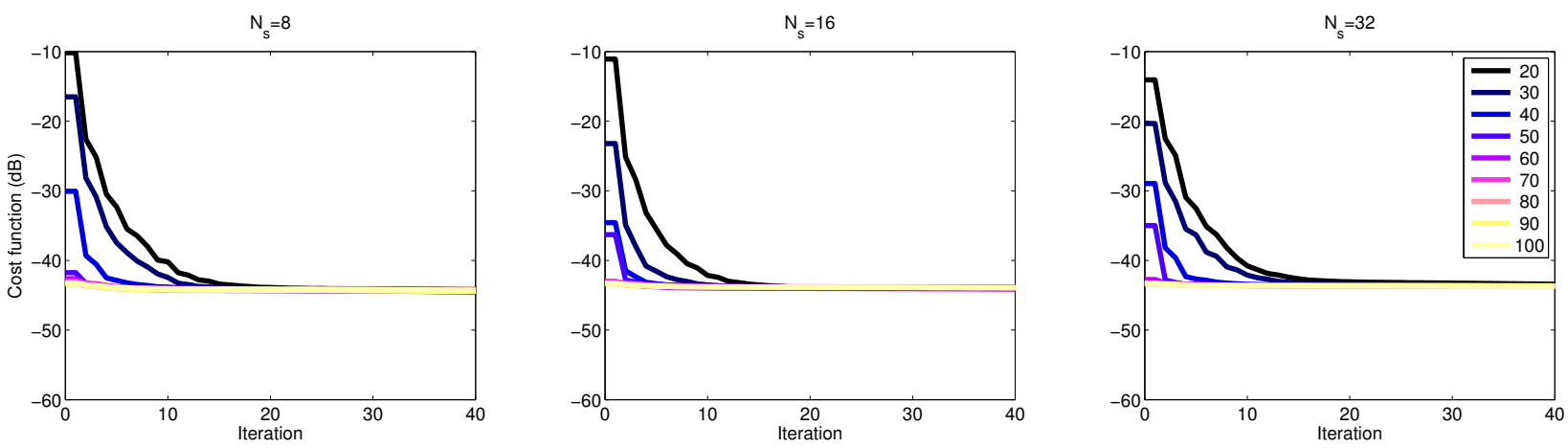

Figure 9. Cost function $\mathcal{F}$ evolution along the iterations for various $\mathrm{SNR}_{m}$ and number of transmitters $N_{s}\left(\mathrm{SNR}_{B}=60 \mathrm{~dB}\right.$, element-based inversion). 


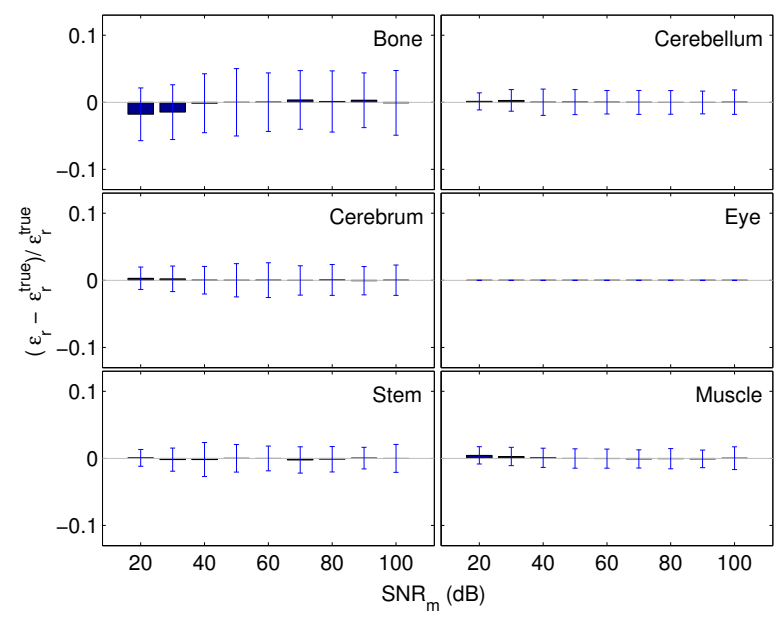

(a) $\mathbf{b}^{\varepsilon_{r}}$ and $\mathbf{s}^{\varepsilon_{r}}, N_{s}=8$

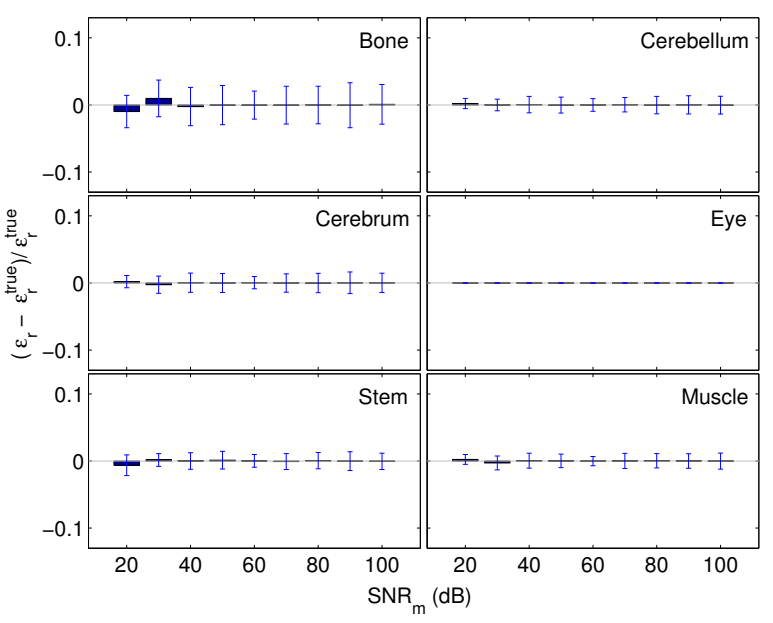

(c) $\mathbf{b}^{\varepsilon_{r}}$ and $\mathbf{s}^{\varepsilon_{r}}, N_{s}=32$

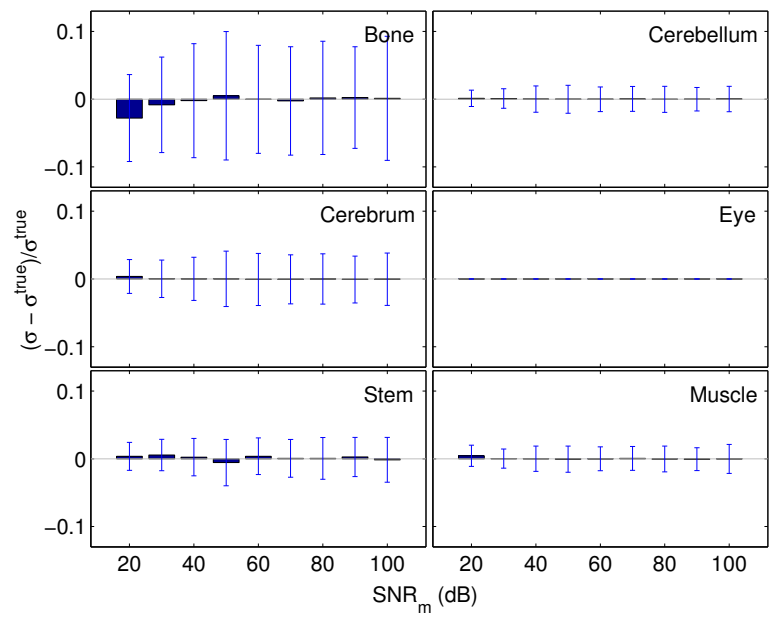

(b) $\mathbf{b}^{\sigma}$ and $\mathbf{s}^{\sigma}, N_{s}=8$

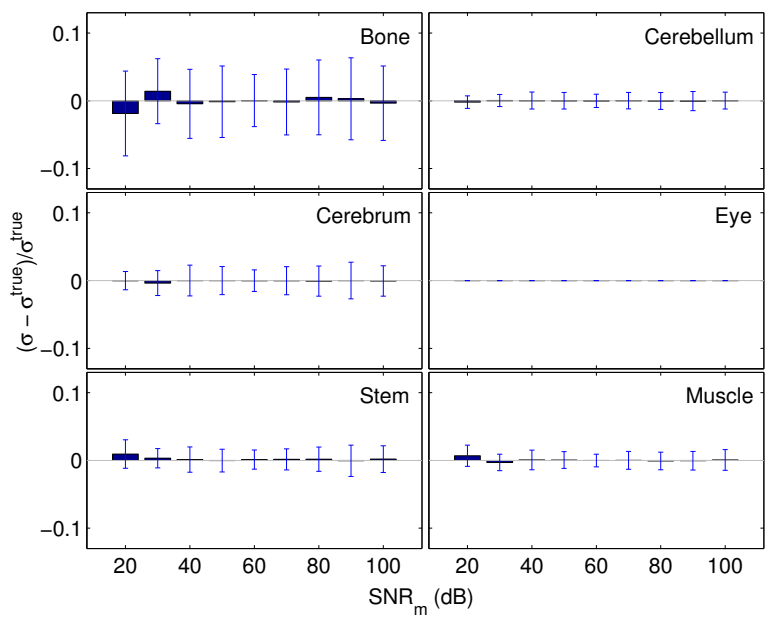

(d) $\mathbf{b}^{\sigma}$ and $\mathbf{s}^{\sigma}, N_{s}=32$

Figure 10. Biases $\mathbf{b}^{\varepsilon_{r}}$ and $\mathbf{b}^{\sigma}$ combined with standard deviations $\mathbf{s}^{\varepsilon_{r}}$ and $\mathbf{s}^{\sigma}$ for various $\mathrm{SNR}_{m}$ and $N_{s}\left(\mathrm{SNR}_{B}=60 \mathrm{~dB}\right.$, element-based inversion $)$.

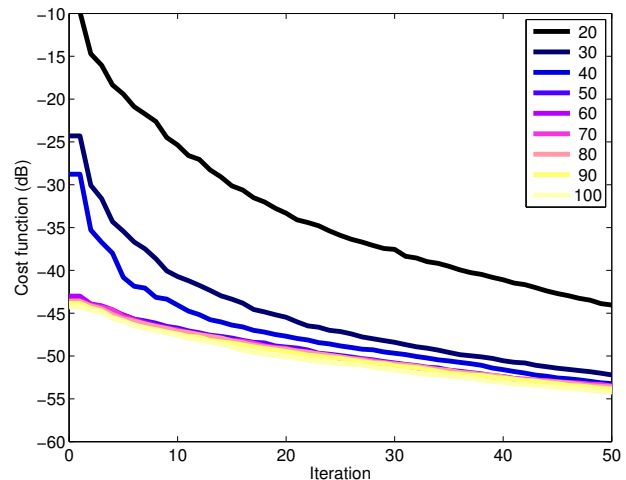

(a) Element-based inversion

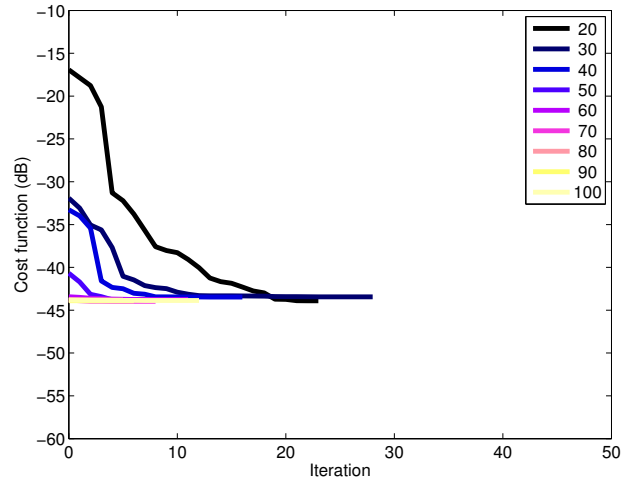

(b) Zone-based inversion

Figure 11. Cost function $\mathcal{F}$ evolution along the iterations for various $\mathrm{SNR}_{m}$ for two different regularization strategies $\left(N_{s}=1, \mathrm{SNR}_{B}=60 \mathrm{~dB}\right)$. 


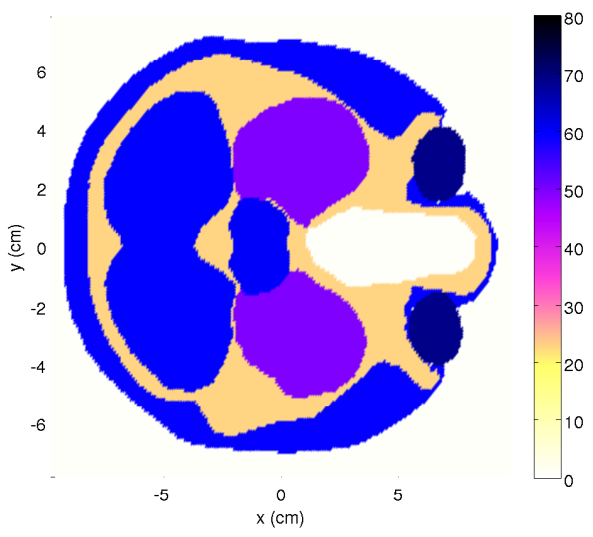

(a) $\mathrm{SNR}_{B}=60 \mathrm{~dB}, \mathrm{SNR}_{m}=40 \mathrm{~dB}$

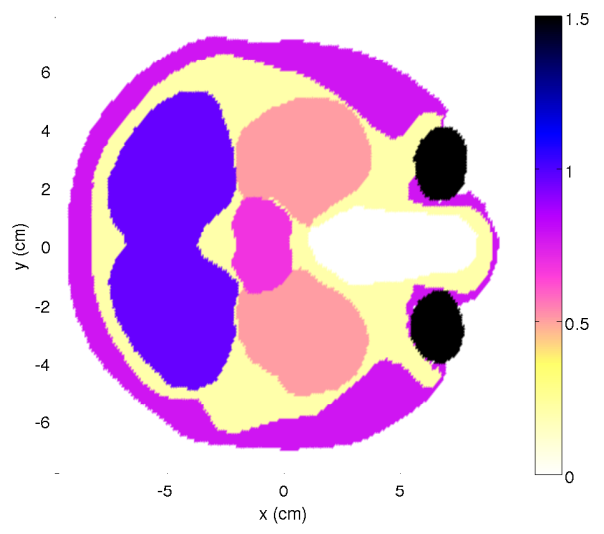

(b) $\mathrm{SNR}_{B}=60 \mathrm{~dB}, \mathrm{SNR}_{m}=40 \mathrm{~dB}$

Figure 12. Final reconstructions: (a) $\varepsilon_{r}=\operatorname{Re}\left(\mathbf{m}^{(*)}\right)$ and (b) $\boldsymbol{\sigma}=\omega \varepsilon_{0} \operatorname{Im}\left(\mathbf{m}^{(*)}\right)$. $\left(N_{s}=1\right.$, zone-based inversion). 